\title{
MHD Radiative Blood Flow Embracing Gold Particles via a Slippery Sheet through an Erratic Heat Sink/Source
}

\author{
Umair Khan ${ }^{1} \mathbb{D}$, Anum Shafiq ${ }^{2} \mathbb{D}$, Aurang Zaib ${ }^{3,4, *}$, El-Sayed M. Sherif ${ }^{5,6} \mathbb{D}$ and \\ Dumitru Baleanu $7,8,9$ \\ 1 Department of Mathematics and Social Sciences, Sukkur IBA University, Sukkur 65200, Sindh Pakistan; \\ umairkhan@iba-suk.edu.pk \\ 2 School of Mathematics and Statistics, Nanjing University of Information Science and Technology, \\ Nanjing 210044, China; anumshafiq@ymail.com \\ 3 Department of Natural Sciences, The Begum Nusrat Bhutto Women University, Sukkur 65170, Pakistan \\ 4 Department of Mathematical Sciences, Federal Urdu University of Arts, Science \& Technology, \\ Gulshan-e-Iqbal Karachi 75300, Pakistan \\ 5 Mechanical Engineering Department, College of Engineering, King Saud University, P.O. Box 800, \\ Al-Riyadh 11421, Saudi Arabia; esherif@ksu.edu.sa \\ 6 Electrochemistry and Corrosion Laboratory, Department of Physical Chemistry, National Research Centre, \\ El-Buhouth St., Dokki, 12622 Cairo, Egypt \\ 7 Department of Mathematics, Cankaya University, 06790 Ankara, Turkey; dumitru.baleanu@gmail.com \\ 8 Institute of Space Sciences, 077125 Magurele, Romania \\ 9 Department of Medical Research, China Medical University Hospital, China Medical University, \\ Taichung 40447, Taiwan \\ * Correspondence: aurangzaib@fuuast.edu.pk
}

Received: 27 July 2020; Accepted: 14 September 2020; Published: 16 September 2020

\begin{abstract}
Cancer remains one of the world's leading healthcare issues, and attempts continue not only to find new medicines but also to find better ways of distributing medications. It is harmful and lethal to most of its patients. The need to selectively deliver cytotoxic agents to cancer cells, to enhance protection and efficacy, has prompted the implementation of nanotechnology in medicine. The latest findings have found that gold nanomaterials can heal and conquer it because the material is studied such as gold (atomic number 79) which produces a large amount of heat and contribute to the therapy of malignant tumors. The purpose of the present study is to research the consequence of heat transport through blood flow (Casson model) that contains gold particles in a slippery shrinking/stretching curved surface. The mathematical modeling of Casson nanofluid containing gold nanomaterials towards the slippery curved shrinking/stretching surface is simplified by utilizing suitable transformation. Numerical dual solutions for the temperature and velocity fields are calculated by using bvp4c methodology in MATLAB. Impacts of related parameters are investigated in the temperature and velocity distribution. The results indicate that the suction parameter accelerates the velocity in the upper branch solution and decelerates it in the lower branch solution, while the temperature diminishes in both solutions. In addition, the Casson parameter shrinks the thickness of the velocity boundary-layer owing to rapid enhancement in the plastic dynamics' viscosity. Moreover, the nanoparticle volume fraction accelerates the viscosity of blood as well as the thermal conductivity. Thus, findings suggested that gold nanomaterials are useful for drug moving and delivery mechanisms since the velocity boundary is regulated by the volume fraction parameter. Gold nanomaterials also raise the temperature field, so that cancer cells can be destroyed.
\end{abstract}

Keywords: MHD (Magnetohydrodynamics) blood flow; Casson fluid; gold particle; non-uniform heat source/sink; thermal radiation; dual solutions 


\section{Introduction}

In the biomedical sciences, gold nanoparticles (GNPs) are significant and emerge as potential factors for treatment. These are treated as carriers of narcotics, contrast agents, radio-infections, and photovoltaic agents. They are also known as efficient drug exchange substances and delivery medicines since they can contain medicinal molecules in large quantities. Gold nanomaterials possess loads of appealing characteristics for cancer treatment usages. They are tiny and thus can reach the whole body. Most significantly, they can join a variety of drugs and proteins, and can effectively attack, weaken, and kill cancer cells. Gold nanostructures have a large atomic amount, which corresponds to heat generation and use in tumor-specific phototherapy. Huang and El-Sayed [1] utilized the gold nanomaterials for the treatment of photothermal cure and in cancer diagnosis. GNPs were considered a significant field of research because of their special, intense Plasmon resonance in observable choice and their usages in biological sciences [2]. In the analysis of their significance, some researchers have investigated the GNPs' flow through two coaxial tubes. The third-grade liquid conveying GNPs in a hollow and a porous tube was investigated through various analytical techniques by researchers $([3,4])$. Recent papers on nanomaterials' and nanoliquids' flows, with different aspects, were cited by [5-10].

Recently, due to its broad use in manufacturing, engineering, and biological processes, the analysis of flow and heat procedures in non-Newtonian liquids has acquired significant attention. The manufacturing of plastic polymers and fiber optics, cosmetic products, and clay coating are some examples of these applications. The legendary Naiver-Stokes equations are not adequate to explain the non-Newtonian fluid attributes; therefore, various physical models including the Ellis and Cross, Carreau, and Casson replicas are required to refill this void. The applications of non-Newtonian liquid stream physical conditions are difficult for investigators because of their complex character and need of constitutive equations to explain all non-Newtonian liquid flow characteristics. Because of assortment in nature flows, it produces various characteristics. Such examples are power-law fluids, Eyring-Powell fluid rheological fluids, Jeffrey fluid, etc. Casson liquid is a closed model of rheological liquid flow to describe properties of non-Newtonian liquid flow of the yield stress. This was developed as a result of the viscous suspension in a liquid flow of cylindrical artifacts while nevertheless, a few fluids that are reportedly well because of their nonlinearity within the fluid flow, yielding stresses, and pseudo-plasticity in nature. Harris [11] and Bird et al. [12] contributed substantially to the exploration of non-Newtonian liquid models through an assortment of rheological properties; later on, many authors $[13,14]$ examined the non-Newtonian flow features with specific flow-control parameters. In recent times, Jamalabadi et al. [15] addressed the transient simulation of the Carreau blood flow via a stenosis artery based on the principles of FHD (Ferrohydrodynamics) and MHD (Magnetohydrodynamics). They indicated that the control of blood temperature can maintain its ideal range of blood temperature. The simulation of fluid-structure interaction of Carreau blood flow through a tiny occlusion in an artery was examined by Amiri et al. [16].

The thermal radiation and magnetic fields with heat transfer containing blood gold nanoparticles become imperative in industrial and biological processes, for instance, lubricants, emulsions, cancer therapy, biofluids in biological polymer and tissue, drug transportation, nuclear fuel slurries, cancer tumor treatment, and bio-medical fluids. Also, the magnetic field is significant, as it is useful to control the blood flow during thermal therapy, or surgery. We, therefore, recommend studying the significance of heat transport with the Casson liquid involving gold GNPs through a curved shrinking/stretching surface. The blood is described by taking a Casson liquid model and Gold nanoparticles are used to destroy bacteria and treatment for cancer in medical operations. Therefore, the mixture of blood with gold nanomaterials ensures that it is acceptable in many medical usages including cancer and catheter treatment. In addition, the thermal radiation (see [17,18]) and non-uniform heat source effects have been included in the study of heat phenomena. Numerical dual solutions have been calculated for both profiles by using bvp4c methodology in MATLAB. 


\section{Problem Formulation}

Consider a 2D steady, incompressible blood flow (Casson fluid) involving gold GNPs through a shrinking/stretched curved bent surface with radius $R$. The coordinates of the curvilinear form $(r, s)$ are to be utilized and the components of velocity corresponding to the curvilinear coordinates are considered as $\left[u_{1}, v_{1}, 0\right]$. The velocity of the shrinking/stretched surface has been presumed along the $s$-direction. The geometry of the problem is depicted in Figure 1. It is also supposed that mass-flux velocity $v_{0}$ with $v_{0}>0$ is recognized as suction and $v_{0}<0$ as an injection, while the constant temperature at the curved surface, and the free stream or ambient temperature, are signified by $T_{w}$ and $T_{\infty}$, respectively. Further, for simplicity, let $r^{a}=r+R$ are the modified space variables. Under these conjectures, along with a boundary-layer approximation, the leading PDEs (partial differential equations) are [19,20]

$$
\begin{gathered}
\frac{\partial}{\partial r}\left[v_{1} r^{a}\right]+\left(r^{a}-r\right) \frac{\partial u_{1}}{\partial s}=0, \\
\frac{\rho_{n f}}{r^{a}} u_{1}^{2}=\frac{\partial p}{\partial r}, \\
v_{1} \frac{\partial u_{1}}{\partial r}+\left(\frac{r^{a}-r}{r^{a}}\right) u_{1} \frac{\partial u_{1}}{\partial s}+\frac{1}{r^{a}} u_{1} v_{1}+\frac{1}{\rho_{n f}}\left(\frac{r^{a}-r}{r^{a}}\right) \frac{\partial p}{\partial s}=-\frac{\sigma_{n f} B^{2}}{\rho_{n f}} u_{1} \\
+\frac{\mu_{n f}}{\rho_{n f}}\left(1+\frac{1}{\gamma}\right)\left(\frac{\partial^{2} u_{1}}{\partial r^{2}}+\frac{1}{r^{a}} \frac{\partial u_{1}}{\partial r}-\frac{u_{1}}{\left(r^{a}\right)^{2}}\right), \\
v_{1} \frac{\partial T}{\partial r}+\left(\frac{r^{a}-r}{r^{a}}\right) u_{1} \frac{\partial T_{1}}{\partial s}=\frac{k_{n f}}{\left(\rho c_{p}\right)_{n f}}\left(\frac{\partial^{2} T_{1}}{\partial r^{2}}+\frac{1}{r^{a}} \frac{\partial T_{1}}{\partial r}\right)-\frac{1}{\left(\rho c_{p}\right)_{n f} r^{a}} \frac{\partial}{\partial r}\left[r^{a} q_{r}\right] \\
+\frac{q^{\prime \prime \prime}}{\left(\rho c_{p}\right)_{n f}}, \quad\left[-\frac{4 \sigma^{*}}{3 k^{*}}\right] \frac{\partial T_{1}^{4}}{\partial r}, T_{1}^{4} \approx-3 T_{\infty}^{4}+4 T_{1} T_{\infty}^{3}, q^{\prime \prime \prime}=\frac{k_{f} u_{w}(s)\left(T_{w}-T_{\infty}\right)}{s v_{f}}\left[A_{1} F^{\prime}+B_{1}\left(\frac{T_{1}-T_{\infty}}{T_{w}-T_{\infty}}\right)\right] .
\end{gathered}
$$

along with the appropriate boundary conditions

$$
\begin{aligned}
& u_{1}=a_{1} \lambda s, v_{1}=v_{0}, T_{1}=T_{w} \text { at } r=0, \\
& u_{1} \rightarrow 0, \frac{\partial u_{1}}{\partial r} \rightarrow 0, T_{1} \rightarrow T_{\infty} \text { as } r \rightarrow \infty
\end{aligned}
$$

where $a_{1}>0$ and $\lambda$ are stretching $(\lambda>0)$ or shrinking $(\lambda<0)$ parameters, $T_{1}$ the temperature, $F^{\prime}$ the velocity, $p$ the pressure, $\sigma^{*}$ the Stefan-Boltzmann constant, $q_{r}$ the radiative heat flux, $q^{\prime \prime \prime}$ the erratic heat sink/source, $B_{1}$ and $A_{1}$ the temperature and space-dependent heat source/sink, $k^{*}$ the mean proportion constant, while the remaining terms are nanofluids in the problem, such as $\rho_{n f}$ the density, $\mu_{n f}$ dynamic viscosity, $\sigma_{n f}$ electrical conductivity, $k_{n f}$ thermal conductivity, and $\left(\rho c_{p}\right)_{n f}$ heat capacitance. The expressions based on the experimental and theoretical results are proposed by Mintsa et al. [21], Afshari et al. [22], and the Makinde and Animasaun [23], and are defined as

$$
\begin{aligned}
& \mu_{n f}=\mu_{f}\left(1+7.3 \phi+123 \phi^{2}\right) \text { for } 0.02<\phi, \rho_{n f}=(1-\phi) \rho_{f}+\phi \rho_{s_{1}}, \\
& \left(\rho c_{p}\right)_{n f}=\left(\rho c_{p}\right)_{f}+\phi\left(\rho c_{p}\right)_{s_{1}}, \frac{k_{n f}}{k_{f}}=\frac{\left(k_{s_{1}}+2 k_{f}\right)-2 \phi\left(k_{f}-k_{s_{1}}\right)}{\left(k_{s_{1}}+2 k_{f}\right)+\phi\left(k_{f}-k_{s_{1}}\right)}, \\
& \frac{\sigma_{n f}}{\sigma_{f}}=\left(1+\frac{3 \phi\left(\frac{\sigma_{s_{1}}}{\sigma_{f}}-1\right)}{\left(\frac{\sigma_{s_{1}}}{\sigma_{f}}+2\right)-\left(\frac{\sigma_{s_{1}}}{\sigma_{f}}-1\right) \phi}\right) .
\end{aligned}
$$


where in the aforementioned Equation (6), the symbols or notations stand for the base fluid and nanofluid, respectively, like $\mu_{f}, \rho_{f}, \rho_{s_{1}}, \sigma_{f}, \sigma_{s_{1}}, k_{f}, k_{s_{1}},\left(\rho c_{p}\right)_{f^{\prime}}\left(\rho c_{p}\right)_{s_{1}}$, which are abbreviated as the viscosity, density, electrical conductivity, thermal conductivity, and the heat capacitance, respectively, and $\phi$ is the volume fraction of nanofluid.
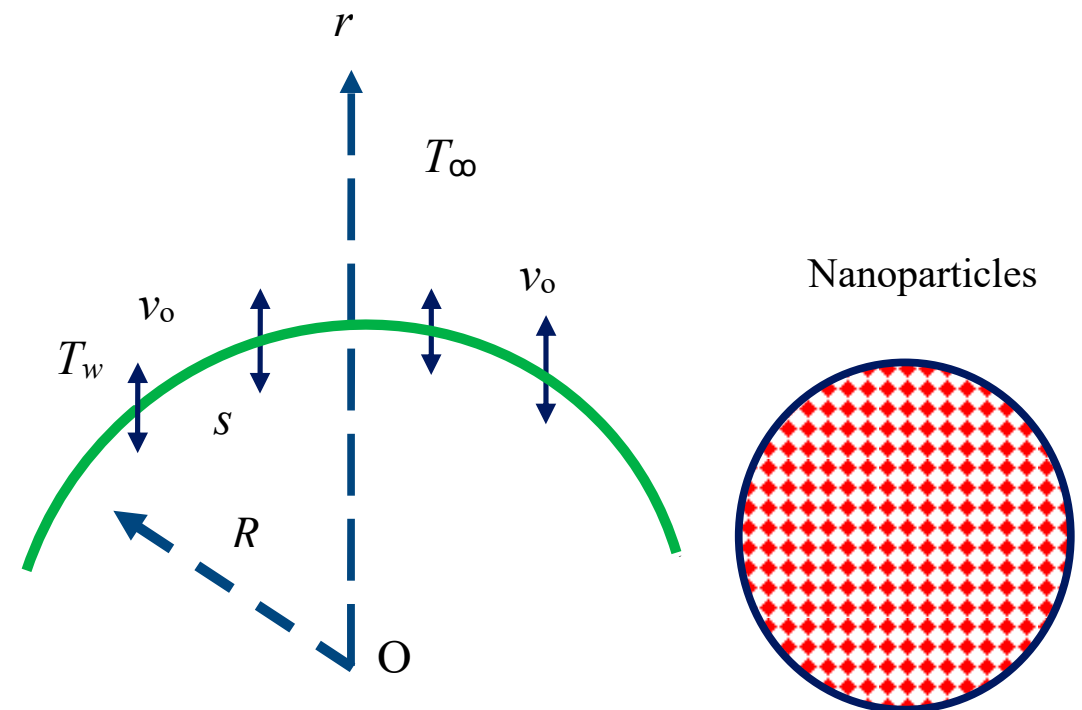

(a) Curved surface

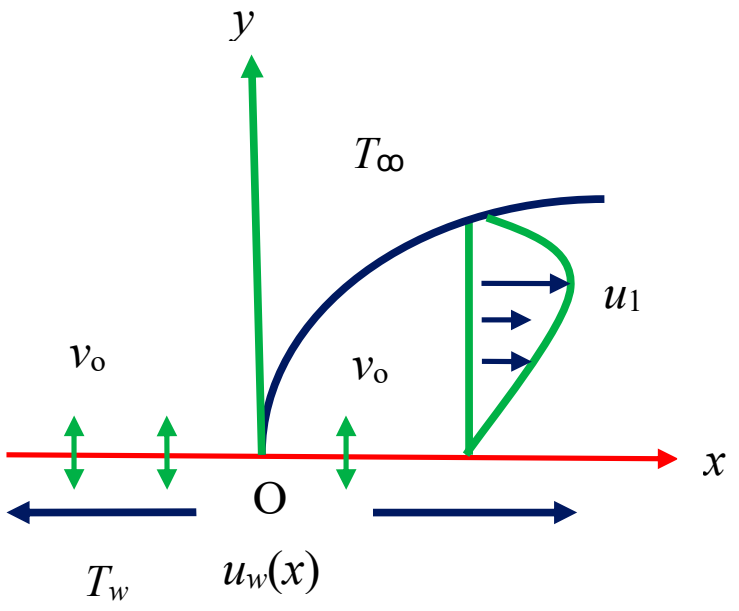

(b) Stretching surface

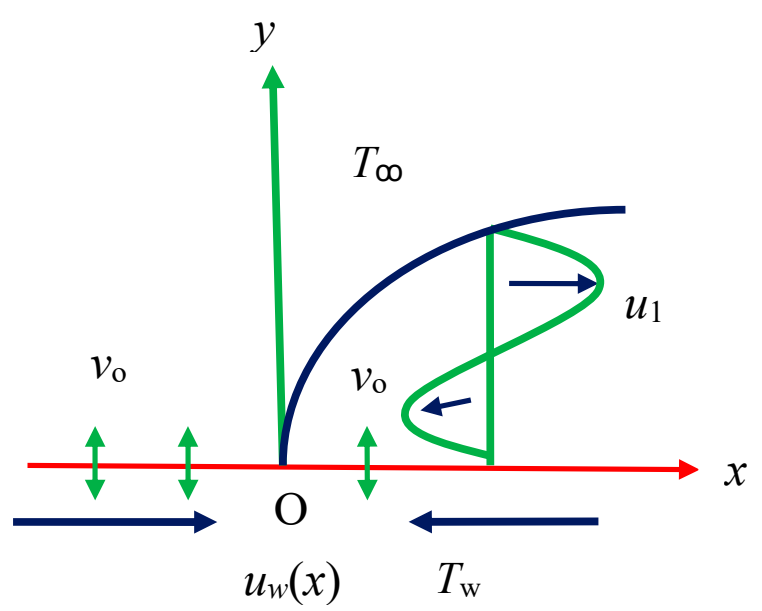

(c) Shrinking surface

Figure 1. (a-c): Physical diagram of the problem.

To further ease this analysis, the transformation is introduced as

$$
\begin{aligned}
& u_{1}=a_{1} s F^{\prime}(\eta), v_{1}=-\left(\frac{r^{a}-r}{r^{a}}\right) \sqrt{a_{1} v_{f}} F(\eta), \theta(\eta)=\frac{T_{1}-T_{\infty}}{T_{w}-T_{\infty}}, \\
& \eta=r \sqrt{\frac{a_{1}}{v_{f}}}, p=\rho_{f} a_{1}^{2} s^{2} P(\eta) .
\end{aligned}
$$

By using Equation (7), the continuity equation is approved, while Equations (2)-(4) suit

$$
\frac{P^{\prime}}{\xi_{1}}=\frac{F^{2}}{\eta+W^{\prime}}
$$




$$
\begin{gathered}
\frac{2 W P}{(\eta+W) \xi_{1}}=\frac{W}{(\eta+W)}\left\{F F^{\prime \prime}-F^{\prime 2}\right\}+\frac{W}{(\eta+W)^{2}} F F^{\prime}-\frac{M}{\xi_{1}} \xi_{3} F^{\prime}+ \\
\left(\frac{\xi_{2}}{\xi_{1}}\right)\left(1+\frac{1}{\gamma}\right)\left\{F^{\prime \prime \prime}+\frac{F^{\prime \prime}}{(\eta+W)}-\frac{F^{\prime}}{(\eta+W)^{2}}\right\}, \\
\left(\xi_{4}+\frac{4}{3} R_{d}\right)\left\{\theta^{\prime \prime}+\frac{\theta^{\prime}}{(\eta+W)}\right\}+\xi_{5} \operatorname{Pr} \frac{W}{(\eta+W)} \theta^{\prime} F+A_{1} F^{\prime}+B_{1} \theta=0,
\end{gathered}
$$

In which:

$$
\begin{aligned}
& \xi_{1}=(1-\phi)+\phi \frac{\rho_{s_{1}}}{\rho_{f}}, \\
& \xi_{2}=1+7.3 \phi+123 \phi^{2}, \\
& \xi_{3}=\left(1+\frac{3 \phi\left(\frac{\sigma_{s_{1}}}{\sigma_{f}}-1\right)}{\left(\frac{\sigma_{s_{1}}}{\sigma_{f}}+2\right)-\left(\frac{\sigma_{s_{1}}}{\sigma_{f}}-1\right) \phi}\right), \\
& \xi_{4}=\frac{\left(k_{s_{1}}+2 k_{f}\right)-2 \phi\left(k_{f}-k_{s_{1}}\right)}{\left(k_{s_{1}}+2 k_{f}\right)+\phi\left(k_{f}-k_{s_{1}}\right)}, \\
& \xi_{5}=(1-\phi)+\phi \frac{\left(\rho c_{p}\right)_{s_{1}}}{\left(\rho c_{p}\right)_{f}},
\end{aligned}
$$

where $M=\sigma_{f} B_{0}^{2} / \rho_{f} a_{1}, \operatorname{Pr}=v_{f} / \alpha_{f}, W=R \sqrt{a_{1} / v_{f}}$ and $R_{d}=4 \sigma^{*} T_{\infty}^{3} / k^{*} k_{f}$ is the magnetic field parameter, Prandtl number, the curvature parameter, and the radiation parameter, respectively.

Eliminating the pressure from Equations (8) and (9), we have

$$
\begin{aligned}
& \left\{\xi_{2}+\frac{\xi_{2}}{\gamma}\right\}\left(F^{(4)}+\frac{2}{\eta+W} F^{\prime \prime \prime}-\frac{1}{(\eta+W)^{2}} F^{\prime \prime}+\frac{1}{(\eta+W)^{3}} F^{\prime}\right)+\frac{\xi_{1} W}{\eta+W}\left(F F^{\prime \prime \prime}-F^{\prime} F^{\prime \prime}\right)- \\
& M \xi_{3}\left(\frac{F^{\prime}}{(\eta+W)}+F^{\prime \prime}\right)-\frac{\xi_{1} W}{(\eta+W)^{2}}\left(F^{2}-F F^{\prime \prime}\right)-\frac{\xi_{1} W}{(\eta+W)^{3}} F F^{\prime}=0 .
\end{aligned}
$$

The boundary restrictions are

$$
\left\{\begin{array}{l}
F(0)=S_{1}, F^{\prime}(0)=\lambda, \theta(0)=1 \text { at } \eta=0, \\
F^{\prime \prime}(\eta) \rightarrow 0, F^{\prime}(\eta) \rightarrow 0, \theta(\eta) \rightarrow 0 \text { as } \eta \rightarrow \infty .
\end{array}\right.
$$

where $S_{1}=-v_{0} / \sqrt{a_{1} v_{f}}$.

From Equation (9), we can calculate the pressure as

$$
P=\frac{(\eta+W) \xi_{1}}{2 W}\left[\begin{array}{l}
\frac{W}{(\eta+W)}\left\{F F^{\prime \prime}-F^{2}\right\}+\frac{W}{(\eta+W)^{2}} F F^{\prime}-\frac{M}{\xi_{1}} \xi_{3} F^{\prime}+ \\
\left(\frac{\xi_{2}}{\xi_{1}}\right)\left(1+\frac{1}{\gamma}\right)\left\{F^{\prime \prime \prime}+\frac{F^{\prime \prime}}{(\eta+W)}-\frac{F^{\prime}}{(\eta+W)^{2}}\right\}
\end{array}\right]
$$

The friction factor coefficient $C_{F}$ and the local Nusselt number $N u_{S}$ are the engineering quantities of interest, which are mathematically written as

$$
C_{F}=\frac{\tau_{s}}{\rho_{f} u_{w}^{2}} \text { and } N u_{s}=\frac{s q_{w}}{k_{f}\left(T_{w}-T_{\infty}\right)} \text { where } q_{w}, \tau_{s} \text { called the wall heat flux and shear stress }
$$


and further, these are defined as

$$
\begin{aligned}
& \tau_{s}=\left.\mu_{n f}\left(1+\frac{1}{\gamma}\right)\left(\frac{\partial u_{1}}{\partial r}-\frac{u_{1}}{r^{a}}\right)\right|_{r=0}, \\
& q_{w}=\left.\left(-k_{n f}\left\{\frac{\partial T_{1}}{\partial r}\right\}+\left(q_{r}\right)_{w}\right)\right|_{r=0} .
\end{aligned}
$$

By plugging Equation (7) in Equations (14) and (15), we get the dimensionless form of skin friction coefficient $C_{F}$ and the local Nusselt number $N u_{S}$ which are given as

$$
\begin{aligned}
& \left\{\operatorname{Re}_{s}\right\}^{0.5} C_{F}=\xi_{2}\left\{1+\frac{1}{\gamma}\right\}\left(F^{\prime \prime}-\frac{F^{\prime}}{\eta+W}\right), \\
& \left\{\operatorname{Re}_{s}\right\}^{-0.5} N u_{s}=-\theta^{\prime}(0)\left\{\xi_{4}+\frac{4}{3} R_{d}\right\}, \quad \text { where } \operatorname{Re}_{s}=a_{1} s^{2} / v_{f} .
\end{aligned}
$$

\section{Methodology}

After removing the pressure, the transformed ODEs (ordinary differential equations) (10) and (11) with the boundary restrictions (12) are solved numerically through the Lobatto IIIA formula. The Lobatto IIIA formula is also signified as a collocation formula with fourth-order accuracy. The mesh selection and control of error is obtained by using the residual of the interrupted solution. In this method, the non-linear system (10)-(12) is rewritten as in the first-order ODEs system by introducing new variables. Let the process can be proceed as follow:

$$
F=Z_{1}^{*}, F^{\prime}=Z_{2}^{*}, F^{\prime \prime}=Z_{3}^{*}, F^{\prime \prime \prime}=Z_{4}^{*}, \theta=Z_{5}^{*}, \theta^{\prime}=Z_{6}^{*}
$$

Inserting the aforementioned new variables in the dimensional form of ODEs will be sealed in the following compact form as

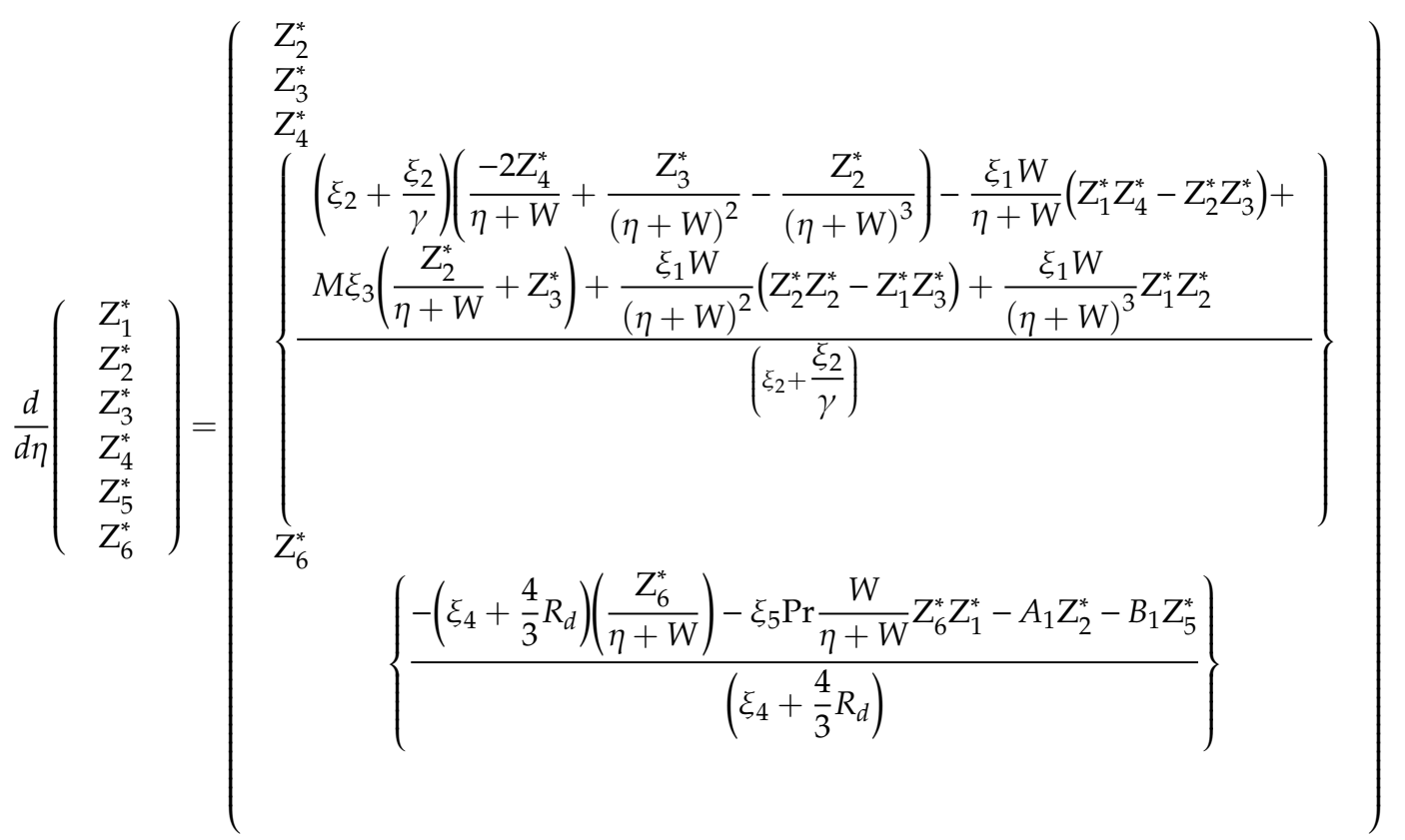

subject to boundary restrictions

$$
\left.\begin{array}{l}
Z_{1}^{*}(0)=S_{1}, Z_{2}^{*}(0)=\lambda, Z_{5}^{*}(0)=1, \\
Z_{2}^{*}(\infty) \rightarrow 0, Z_{3}^{*}(\infty) \rightarrow 0, Z_{5}^{*}(\infty) \rightarrow 0 .
\end{array}\right\}
$$


The preceding equations' scheme may probably have different solutions or more than two solutions. The provided numerical solution, therefore, required specific early guesses to fulfill the conditions (19). Therefore, some initial early guesses are needed to operate the bvp4c process. The initial or early guess is quite simple when it comes to finding the first solution compared to achieving the second solution, as they needed the best suitable guess, which is quite difficult. The maximum range of numerical integration is considered as $\eta_{\max }=2$ in the simulation analysis, which is very useful for asymptotically obeying the conditions in the appropriate or schematic solutions. The mesh size value is considered $\Delta \eta=0.01$. The iterative process is repeated, waiting for the necessary results to be obtained to match the convergence criterion up to the precision point $10^{-5}$.

\section{Stability Analysis}

The literature review generally offers a greater number of insightful works concerned with the analysis of the stability of the fluid flows such as the boundary layer, in which the researchers tested the adequacy of more than one solution (multiple) extracted from a physical point of view and rejected the unstable ones (which were not physically realizable). In this regard, the readers can refer to numerous research studies stated by Weidman et al. [24], Zaib et al. [25], Sharma et al. [26], and Rosca and Pop [27]. Based on these references, they have investigated that the solution of the first branch is physically realizable (stable) and the solution of the second branch is not realizable (unstable) in physical practice. To determine the physical significance of these solutions, we consider the mathematical problem in the unsteadiness form as given below:

$$
\begin{gathered}
\frac{\partial}{\partial r}\left[v_{1} r^{a}\right]+\left(r^{a}-r\right) \frac{\partial u_{1}}{\partial s}=0 \\
\frac{\rho_{n f}}{r^{a}} u_{1}^{2}=\frac{\partial p}{\partial r^{\prime}} \\
\frac{\partial u_{1}}{\partial t}+v_{1} \frac{\partial u_{1}}{\partial r}+\left(\frac{r^{a}-r}{r^{a}}\right) u_{1} \frac{\partial u_{1}}{\partial s}+\frac{1}{r^{a}} u_{1} v_{1}+\frac{1}{\rho_{n f}}\left(\frac{r^{a}-r}{r^{a}}\right) \frac{\partial p}{\partial s}=-\frac{\sigma_{n f} B^{2}}{\rho_{n f}} u_{1} \\
+\frac{\mu_{n f}}{\rho_{n f}}\left(1+\frac{1}{\gamma}\right)\left(\frac{\partial^{2} u_{1}}{\partial r^{2}}+\frac{1}{r^{a}} \frac{\partial u_{1}}{\partial r}-\frac{u_{1}}{\left(r^{a}\right)^{2}}\right), \\
\frac{\partial T}{\partial t}+v_{1} \frac{\partial T}{\partial r}+\left(\frac{r^{a}-r}{r^{a}}\right) u_{1} \frac{\partial T_{1}}{\partial s}=\frac{k_{n f}}{\left(\rho c_{p}\right)_{n f}}\left(\frac{\partial^{2} T_{1}}{\partial r^{2}}+\frac{1}{r^{a}} \frac{\partial T_{1}}{\partial r}\right)-\frac{1}{\left(\rho c_{p}\right)_{n f} r^{\prime}} \frac{\partial}{\partial r}\left[r^{a} q_{r}\right] \\
q_{r}^{\prime \prime \prime}, \quad\left[-\frac{4 \sigma^{*}}{3 k^{*}}\right) \frac{\partial T_{1}^{4}}{\partial r}, T_{1}^{4} \approx-3 T_{\infty}^{4}+4 T_{1} T_{\infty}^{3}, q^{\prime \prime \prime}=\frac{k_{f} u_{w}(s)\left(T_{w}-T_{\infty}\right)}{s v_{f}}\left[A_{1} f^{\prime}+B_{1}\left(\frac{T_{1}-T_{\infty}}{T_{w}-T_{\infty}}\right)\right] .
\end{gathered}
$$

along with the appropriate boundary conditions

$$
\begin{aligned}
& u_{1}=\frac{a_{1} \lambda s}{1-\beta t^{\prime}}, v_{1}=v_{w}, T_{1}=T_{w} \text { at } r=0, \\
& u_{1} \rightarrow 0, \frac{\partial u_{1}}{\partial r} \rightarrow 0, T_{1} \rightarrow T_{\infty} \text { as } r \rightarrow \infty .
\end{aligned}
$$

Here, for the unsteady problem, we take the velocity at the boundary of the curved surface in terms of time such as $u_{1}=\frac{a_{1} \lambda s}{1-\beta t}$ where $\beta<0$ is signify the decelerated sheet and $\beta>0$ is used for the accelerated sheet, while the rest of them are discussed in the mathematical formulation. On the other hand, the porosity of the sheet is taken to be variable and is defined as $v_{w}(t)=-\sqrt{\frac{a_{1} v_{f}}{1-\beta t}} S_{1}$, where $S_{1}$ 
represents the uniform wall mass suction parameter, with $S_{1}<0$ representing the case of injection or blowing and $S_{1}>0$ denote the phenomenon of suction, respectively. To further ease this analysis, we introduce the new transformation along with the dimensionless variable $\tau$ for the aforementioned unsteady problem, such as:

$$
\begin{aligned}
& u_{1}=\frac{a_{1} s}{1-\beta t} \frac{\partial F(\eta, \tau)}{\partial \eta}, v_{1}=-\left(\frac{r^{a}-r}{r^{a}}\right) \sqrt{\frac{a_{1} v_{f}}{1-\beta t}} F(\eta, \tau), \theta=\frac{T_{1}(\eta, \tau)-T_{\infty}}{T_{w}-T_{\infty}} \\
& \eta=r \sqrt{\frac{a_{1}}{v_{f} 1-\beta t}}, p=\frac{\rho_{f} a_{1}{ }^{2} s^{2}}{(1-\beta t)^{2}} P(\eta, \tau), \tau=a_{1} t
\end{aligned}
$$

where the coordinate $t$ elucidates the dimensional temporal variable. Plugging Equation (25), the continuity is identically true, while Equations (21) to (23) take the form of:

$$
\begin{gathered}
\frac{\partial P}{\partial \eta}=\frac{\xi_{1}}{\eta+W}\left(\frac{\partial F}{\partial \eta}\right)^{2} \\
\frac{2 W P}{(\eta+W) \xi_{1}}=\frac{W}{(\eta+W)}\left\{F \frac{\partial^{2} F}{\partial \eta^{2}}-\left(\frac{\partial F}{\partial \eta}\right)^{2}\right\}+\frac{W}{(\eta+W)^{2}} F \frac{\partial F}{\partial \eta}-\frac{M}{\xi_{1}} \xi_{3} \frac{\partial F}{\partial \eta}+ \\
\left(\frac{\xi_{2}}{\xi_{1}}\right)\left(1+\frac{1}{\gamma}\right)\left\{\frac{\partial^{3} F}{\partial \eta^{3}}+\frac{\frac{\partial^{2} F}{\partial \eta^{2}}}{(\eta+W)}-\frac{\frac{\partial F}{\partial \eta}}{(\eta+W)^{2}}\right\}-B\left(\frac{\partial F}{\partial \eta}+\frac{\eta}{2} \frac{\partial^{2} F}{\partial \eta^{2}}\right)-(1-\beta t) \frac{\partial^{2} F}{\partial \eta \partial \tau} \\
\left(\xi_{4}+\frac{4}{3} R_{d}\right)\left\{\frac{\partial^{2} \theta}{\partial \eta^{2}}+\frac{1}{(\eta+W)} \frac{\partial \theta}{\partial \eta}\right\}+\xi_{5} \operatorname{Pr}\left(\frac{W}{(\eta+W)} F \frac{\partial \theta}{\partial \eta}-B \frac{\eta}{2} \frac{\partial \theta}{\partial \eta}-(1-\beta t) \frac{\partial \theta}{\partial \tau}\right)+ \\
A_{1} F^{\prime}+B_{1} \theta=0 .
\end{gathered}
$$

Here, involved dimensional constraints such as $W=R \sqrt{a_{1} / v_{f}(1-\beta t)}$ and $B=\beta / a_{1}$ are called the curvature parameter and the unsteadiness parameter, respectively.

Eliminating the pressure from Equations (26) and (27), we have

$$
\begin{aligned}
& \left\{\xi_{2}+\frac{\xi_{2}}{\gamma}\right\}\left(\frac{\partial^{4} F}{\partial \eta^{4}}+\frac{2}{\eta+W} \frac{\partial^{3} F}{\partial \eta^{3}}-\frac{1}{(\eta+W)^{2}} \frac{\partial^{2} F}{\partial \eta^{2}}+\frac{1}{(\eta+W)^{3}} \frac{\partial F}{\partial \eta}\right)-\frac{\xi_{1} W}{\eta+W}\left(\frac{\partial F}{\partial \eta} \frac{\partial^{2} F}{\partial \eta^{2}}-F \frac{\partial^{3} F}{\partial \eta^{3}}\right)- \\
& \frac{\xi_{1} W}{(\eta+W)^{2}}\left(\left(\frac{\partial F}{\partial \eta}\right)^{2}-F \frac{\partial^{2} F}{\partial \eta^{2}}\right)-\frac{\xi_{1} W}{(\eta+W)^{3}} F \frac{\partial F}{\partial \eta}-M \xi_{3}\left(\frac{1}{(\eta+W)} \frac{\partial F}{\partial \eta}+\frac{\partial^{2} F}{\partial \eta^{2}}\right)- \\
& \frac{B}{\eta+W} \xi_{1}\left(\frac{\partial F}{\partial \eta}+\frac{\eta}{2} \frac{\partial^{2} F}{\partial \eta^{2}}\right)-\frac{B}{2} \xi_{1}\left(3 \frac{\partial^{2} F}{\partial \eta^{2}}+\eta \frac{\partial^{3} F}{\partial \eta^{3}}\right)-\frac{(1-\beta t)}{\eta+W} \xi_{1} \frac{\partial^{2} F}{\partial \eta \partial \tau}-(1-\beta t) \xi_{1} \frac{\partial^{3} F}{\partial \eta^{2} \partial \tau}=0 .
\end{aligned}
$$

Along with the subjected boundary restrictions, which are

$$
\left\{\begin{array}{l}
\frac{\partial F}{\partial \eta}(0, \tau)=\lambda, F(0, \tau)=S_{1}, \theta(0, \tau)=1 \text { at } \eta=0, \\
\frac{\partial^{2} F}{\partial \eta^{2}}(\eta, \tau) \rightarrow 0, \frac{\partial F}{\partial \eta}(\eta, \tau) \rightarrow 0, \theta(\eta, \tau) \rightarrow 0 \text { as } \eta \rightarrow \infty .
\end{array}\right.
$$

To investigate the stability process of the steady flow outcomes $F(\eta)=F_{0}(\eta)$ and $\theta(\eta)=\theta_{0}(\eta)$ satisfying the boundary value problem (10), (11) and (12), we can write (see Weidman et al. [24])

$$
\left\{\begin{array}{l}
F(\eta, \tau)=F_{0}(\eta)+e^{-\varepsilon \tau} H(\eta, \tau) \\
\theta(\eta, \tau)=\theta_{0}(\eta)+e^{-\varepsilon \tau} G(\eta, \tau)
\end{array}\right.
$$


where $\varepsilon$ is an unknown eigenvalue parameter, $F(\eta, \tau)$ and $\theta(\eta, \tau)$ are small relative to $F_{0}(\eta)$ and $\theta_{0}(\eta)$. Substituting Equation (31) into Equations (28) to (29), we get the following linearized problem:

$$
\begin{aligned}
& \left\{\xi_{2}+\frac{\xi_{2}}{\gamma}\right\}\left(\frac{\partial^{4} H}{\partial \eta^{4}}+\frac{2}{\eta+W} \frac{\partial^{3} H}{\partial \eta^{3}}-\frac{1}{(\eta+W)^{2}} \frac{\partial^{2} H}{\partial \eta^{2}}+\frac{1}{(\eta+W)^{3}} \frac{\partial H}{\partial \eta}\right)- \\
& \frac{\xi_{1} W}{\eta+W}\left(F_{0}^{\prime \prime} \frac{\partial H}{\partial \eta}+F_{0} \frac{\partial^{2} H}{\partial \eta^{2}}-F_{0} \frac{\partial^{3} H}{\partial \eta^{3}}-F_{0}^{\prime \prime \prime} H\right)+\frac{\xi_{1} W}{(\eta+W)^{2}}\left(F_{0}^{\prime \prime} H-2 F_{0} H-F_{0} \frac{\partial^{2} H}{\partial \eta^{2}}\right) \\
& -\frac{\xi_{1} W}{(\eta+W)^{3}}\left(F_{0} \frac{\partial H}{\partial \eta}+H F_{0}{ }^{\prime}\right)-M \xi_{3}\left(\frac{1}{(\eta+W)} \frac{\partial H}{\partial \eta}+\frac{\partial^{2} H}{\partial \eta^{2}}\right)- \\
& \frac{B}{\eta+W} \xi_{1}\left(\frac{\partial H}{\partial \eta}+\frac{\eta}{2} \frac{\partial^{2} H}{\partial \eta^{2}}\right)-\frac{B}{2} \xi_{1}\left(3 \frac{\partial^{2} H}{\partial \eta^{2}}+\eta \frac{\partial^{3} H}{\partial \eta^{3}}\right)+\frac{(1-\beta t)}{\eta+W} \xi_{1}\left(\varepsilon \frac{\partial H}{\partial \eta}-\frac{\partial^{2} H}{\partial \eta \partial \tau}\right) \\
& +(1-\beta t) \xi_{1}\left(\varepsilon \frac{\partial^{2} H}{\partial \eta^{2}}-\frac{\partial^{3} H}{\partial \eta^{2} \partial \tau}\right)=0 . \\
& \left(\xi_{4}+\frac{4}{3} R_{d}\right)\left\{\frac{\partial^{2} G}{\partial \eta^{2}}+\frac{1}{(\eta+W)} \frac{\partial G}{\partial \eta}\right\}+\xi_{5} \operatorname{Pr}\left(\begin{array}{l}
\frac{W}{(\eta+W)}\left(F_{0} \frac{\partial G}{\partial \eta}+H \theta_{0}{ }^{\prime}\right)-B \frac{\eta}{2} \frac{\partial G}{\partial \eta}+ \\
(1-\beta t)\left(\varepsilon G-\frac{\partial G}{\partial \tau}\right)
\end{array}\right)+ \\
& A_{1} \frac{\partial H}{\partial \eta}+B_{1} G=0,
\end{aligned}
$$

along with the subjected boundary restrictions, which are follow as:

$$
\left\{\begin{array}{l}
\frac{\partial H}{\partial \eta}(0, \tau)=0, H(0, \tau)=0, G(0, \tau)=0, \\
\frac{\partial^{2} H}{\partial \eta^{2}}(\eta, \tau) \rightarrow 0, \frac{\partial H}{\partial \eta}(\eta, \tau) \rightarrow 0, G(\eta, \tau) \rightarrow 0 \text { as } \eta \rightarrow \infty .
\end{array}\right.
$$

Furthermore, we tested the process of the stability analysis of the time-independent flow outcomes such as $F_{0}(\eta)$ and $\theta_{0}(\eta)$ by setting the dimensional new variable $\tau=0$ in Equations (32) to (33), along with the boundary conditions (34), and hence $H=H_{0}(\eta)$ and $G=G_{0}(\eta)$ involved in the aforesaid reference equations, which demonstrates the initial decay or growth of the outcomes (31). Due to this fact, we have to tackle the following linear eigenvalue problem

$$
\begin{aligned}
& \left\{\xi_{2}+\frac{\xi_{2}}{\gamma}\right\}\left(H_{0}^{\prime \prime \prime \prime}+\frac{2}{\eta+W} H_{0}^{\prime \prime \prime}-\frac{1}{(\eta+W)^{2}} H_{0}^{\prime \prime}+\frac{1}{(\eta+W)^{3}} H_{0}{ }^{\prime}\right)- \\
& \frac{\xi_{1} W}{\eta+W}\left(F_{0}^{\prime \prime} H_{0}{ }^{\prime}+F_{0}{ }^{\prime} H_{0}^{\prime \prime}-F_{0} H_{0}^{\prime \prime \prime}-F_{0}^{\prime \prime \prime} H_{0}\right)+\frac{\xi_{1} W}{(\eta+W)^{2}}\left(F_{0}{ }^{\prime \prime} H_{0}-2 F_{0} H_{0}-F_{0} H_{0}{ }^{\prime \prime}\right) \\
& -\frac{\xi_{1} W}{(\eta+W)^{3}}\left(F_{0} H_{0}{ }^{\prime}+H_{0} F_{0}{ }^{\prime}\right)-M \xi_{3}\left(\frac{1}{(\eta+W)} H_{0}{ }^{\prime}+H_{0}^{\prime \prime}\right)- \\
& \frac{B}{\eta+W} \xi_{1}\left(H_{0}{ }^{\prime}+\frac{\eta}{2} H_{0}^{\prime \prime}\right)-\frac{B}{2} \xi_{1}\left(3 H_{0}{ }^{\prime \prime}+\eta H_{0}^{\prime \prime \prime}\right)+\frac{\xi_{1} \varepsilon H_{0}{ }^{\prime}}{\eta+W}+\xi_{1} \varepsilon H_{0}{ }^{\prime \prime}=0 . \\
& \left(\xi_{4}+\frac{4}{3} R_{d}\right)\left\{G_{0}{ }^{\prime \prime}+\frac{G_{0}{ }^{\prime}}{(\eta+W)}\right\}+\xi_{5} \operatorname{Pr}\left(\frac{W}{(\eta+W)}\left(F_{0} G_{0}{ }^{\prime}+H_{0} \theta_{0}{ }^{\prime}\right)-B \frac{\eta}{2} G_{0}{ }^{\prime}+\varepsilon G_{0}\right)+ \\
& A_{1} H_{0}{ }^{\prime}+B_{1} G_{0}=0,
\end{aligned}
$$

along with the subjected boundary restrictions, which are follow as

$$
\left\{\begin{array}{l}
H_{0}{ }^{\prime}(0)=0, H_{0}(0)=0, G_{0}(0)=0, \\
H_{0}{ }^{\prime \prime}(\eta) \rightarrow 0, H_{0}{ }^{\prime}(\eta) \rightarrow 0, G_{0}(\eta) \rightarrow 0 \text { as } \eta \rightarrow \infty .
\end{array}\right.
$$


It should be specified here that for the particular involved dimensional constraints that have fixed values in the problem, the stability of the time-independent flow solutions $F_{0}(\eta)$ and $\theta_{0}(\eta)$ are determined by the smallest eigenvalue $\varepsilon$. Thus, in accordance with Zaib et al. [25], and Rosca and Pop [27], where they have investigated the relaxing boundary condition on $H_{0}(\eta)$ or $G_{0}(\eta)$ to determine the possible range of all eigenvalues. Hence, in this problem, we relax the condition that $H_{0}{ }^{\prime \prime}(\eta) \rightarrow 0$ as $\eta \rightarrow \infty$, and for a fixed value of $\varepsilon$, Equations (35) to (36) along with the new boundary condition $H_{0}^{\prime \prime \prime}(\eta)=1$ are to be solved.

Moreover, outcomes of the eigenvalue problem (35), (36) and (37) give an infinite number of eigenvalues $\varepsilon_{1}<\varepsilon_{2}<\varepsilon_{3}<\varepsilon_{4}<\ldots \ldots$. obtained when the boundary condition $H_{0}{ }^{\prime \prime}(\eta) \rightarrow 0$ as $\eta \rightarrow \infty$ is satisfied. It is worth mentioning that the smallest eigenvalue $\varepsilon_{1}$ can take positive or negative values. Thus, for the case of the shrinking sheet $(\lambda<0)$ where dual solutions of Equations (10) to (11) subject to the boundary condition (12) exist, the solutions (35) to (36) go to $F_{0}(\eta)$ and $\theta_{0}(\eta)$ when $\varepsilon_{1}>0$ and $\tau \rightarrow \infty$ (steady-state solution), so that there is an initial decay and the first branch solution is stable and physically realizable. However, for $\varepsilon_{1}<0$, there is an initial growth of disturbances, so that the lower branch solution blows up when $\tau \rightarrow \infty$ and, therefore, this solution is not stable and hence not physically realizable.

\section{Results and Discussion}

In this section, the outcomes are managed through conveying the values of emerging constraints as $M=0.01, W=0.5, R_{d}=02, \lambda=-2, S_{1}=2.2, \phi=0.03, \gamma=0.1, A_{1}=B_{1}=0.5$ except the values mentioned in the portraits, whereas the value of the Prandtl number is considered as 21 [28,29], keeping in mind that the base liquid is blood. Table 1 describes the thermo-physical properties of blood and nanoparticles. Table 2 conveys the appraisal of the present solutions for skin factor with varied values of $W$ through the accessible results of [30,31]. In addition, Figure 2 is portrayed to check the current numerical method graphically with the outcomes of [31] in the limited case and found an excellent synchronization between the current and available graphical outcomes. It established an admirable concurrence through their consequences. In the illustrations, the dashed red lines imply the lower branch (LBS) solution, and solid green lines define the upper branch (UBS) solution. In the entire graphs, $\theta(\eta)$ and $F^{\prime}(\eta)$ stimulate the temperature and velocity fields.

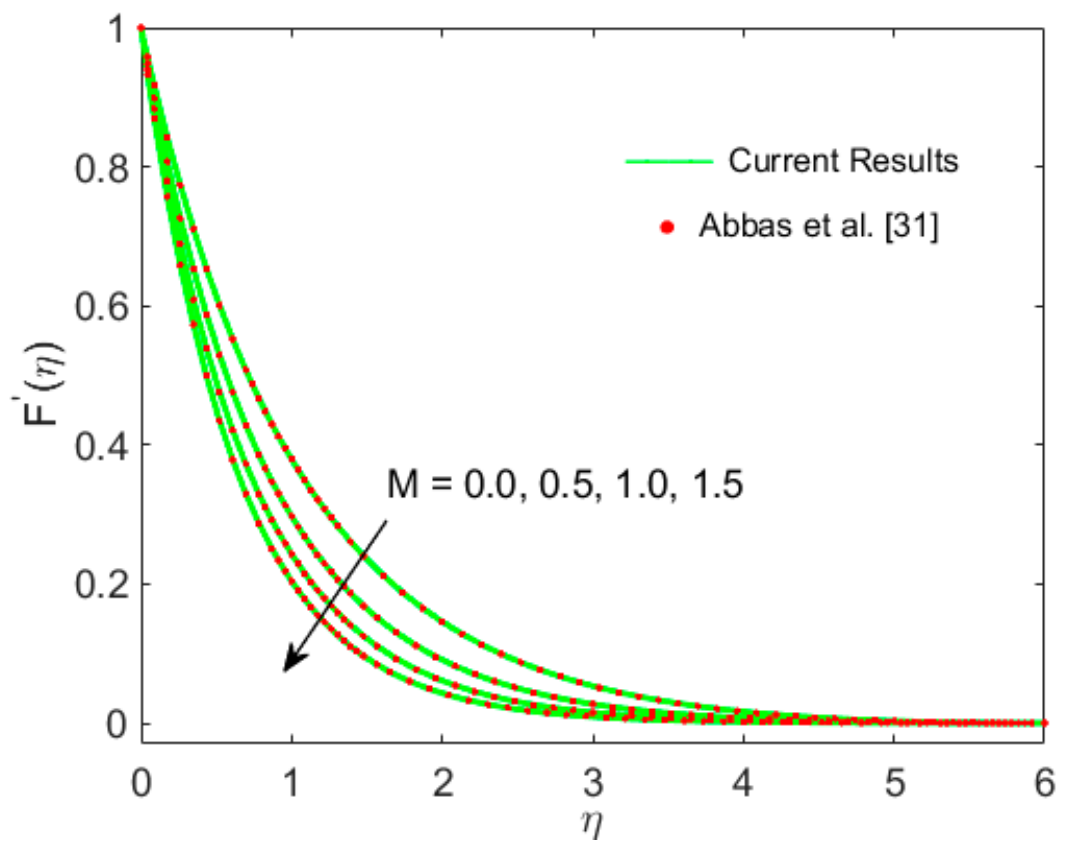

Figure 2. Comparison of the velocity profile $F^{\prime}(\eta)$ for different values of $M$ when $\gamma=\infty, \phi_{1}=\phi_{2}=S_{1}=0$, $\lambda=1, W=10$. 
Table 1. Thermophysical properties of blood and gold nanoparticle [32].

\begin{tabular}{ccc}
\hline Thermophysical Properties & Blood & Gold \\
\hline$c_{p}(\mathrm{~J} / \mathrm{kgK})$ & 3617 & 129 \\
\hline$\rho\left(\mathrm{kg} / \mathrm{m}^{3}\right)$ & 1050 & 19,300 \\
\hline$\sigma(\mathrm{S} / \mathrm{m})$ & 1090 & $4.1 \times 10^{6}$ \\
\hline$k(\mathrm{~W} / \mathrm{mK})$ & 0.52 & 318 \\
\hline
\end{tabular}

Table 2. Present outcomes comparison of the skin factor when $\gamma \rightarrow \infty, S_{1}=M=0, \phi=0$.

\begin{tabular}{cccc}
\hline $\boldsymbol{W}$ & Sanni et al. [30] & Abass et al. [31] & Current Results \\
\hline 5.0 & 1.15076 & 1.1576 & 1.1507664 \\
\hline 10.0 & 1.0734 & 1.0735 & 1.0734886 \\
\hline 20.0 & 1.0355 & 1.0356 & 1.0356098 \\
\hline 30.0 & 1.0235 & 1.0235 & 1.0235311 \\
\hline 40.0 & 1.0176 & 1.0176 & 1.0175866 \\
\hline 50.0 & 1.0140 & 1.0141 & 1.0140492 \\
\hline 100.0 & 1.0070 & 1.0070 & 1.0070384 \\
\hline 200.0 & 1.0036 & 1.0036 & 1.0035642 \\
\hline 1000.0 & 1.0008 & 1.0008 & 1.0007993 \\
\hline$\infty$ & 1.0000 & 1.0000 & 1.0000000
\end{tabular}

\subsection{Velocity Distribution Behavior}

Here, the variations of emerging parameters $S_{1}, W, \lambda, \gamma, M$ and as well as $\phi$ on $F^{\prime}(\eta)$ are analyzed. In this regard, Figures 3-8 are prepared. Figure 3 depicts the trend of $F^{\prime}(\eta)$ for diverse values of $S_{1}$. This Figure perceives that the velocity augments with $S_{1}$ in the UBS and shrinks in the LBS. Physically, the confrontation in the blood flow is happening due to viscosity, which can be managed through the suction. Elevating the suction leads to the drag force reduction in the surface. The effect of $W$ on the flow velocity profile is presented in Figure 4. The liquid velocity is enhanced due to greater values of $W$ in both the UBS and LBS. Physically, the values of $W$ permit us to envisage that less kinematic viscous difficulty will have occurred for an increased curvature parameter. Therefore, the blood flow moves smoothly. The impact of $M$ on $F^{\prime}(\eta)$ can be observed in Figure 5. The fluid velocity $F^{\prime}(\eta)$ decays with uplifting values of $M$ in both results. Physically, a greater amount of magnetic field generates a type of resistive force in the flow, which behaves against the flow path. Because of this, the blood velocity is superior. This also discloses an imperative inspection that the blood motion in a moving vessel of blood can be regulated through concerning an external magnetic field and varying the strength of the pertained field. The impact of the Casson parameter $\gamma$ on $F^{\prime}(\eta)$ is sketched in Figure 6. It is transparent to observe the behavior of liquid velocity, which is augmented owing to $\gamma$ in the UBS and during the LBS, and which consequently shrinks the velocity boundary-layer. Physically, the augmenting in the Casson constraint leads to a fall in the blood plasticity, and as an output, the thickness of the velocity boundary shrinks. Figure 7 suggests that the velocity is elevating the function of the nanoparticle volume fraction in both results and, as a consequence, the thickness of the velocity boundary decelerates. Physically, an augmentation in the volume nanoparticle fraction leads to an improvement in the viscosity of blood, which in turn the shrinks the velocity thickness of the boundary-layer. The influence of the shrinking parameter $\lambda$ on the velocity profile $F^{\prime}(\eta)$ is shown in Figure 8 . The velocity gradient and also the thickness of the momentum boundary layer is decelerated in the UBS and accelerated in the LBS as we boost up the values of the shrinking parameter. Moreover, the gap between the curves in the UBS is lesser as compared to the solution curves in the LBS. 


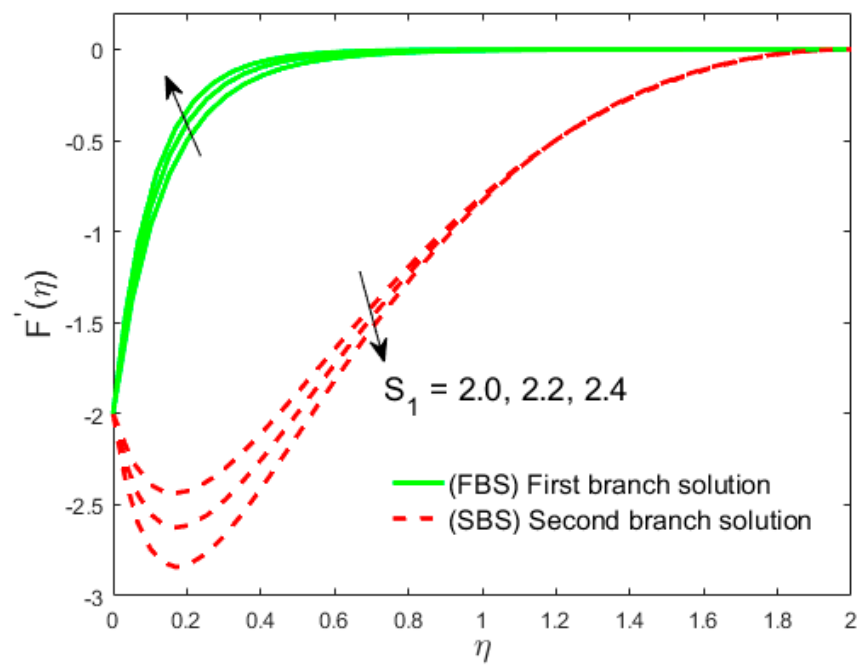

Figure 3. The impact of $S_{1}$ on $F^{\prime}(\eta)$.

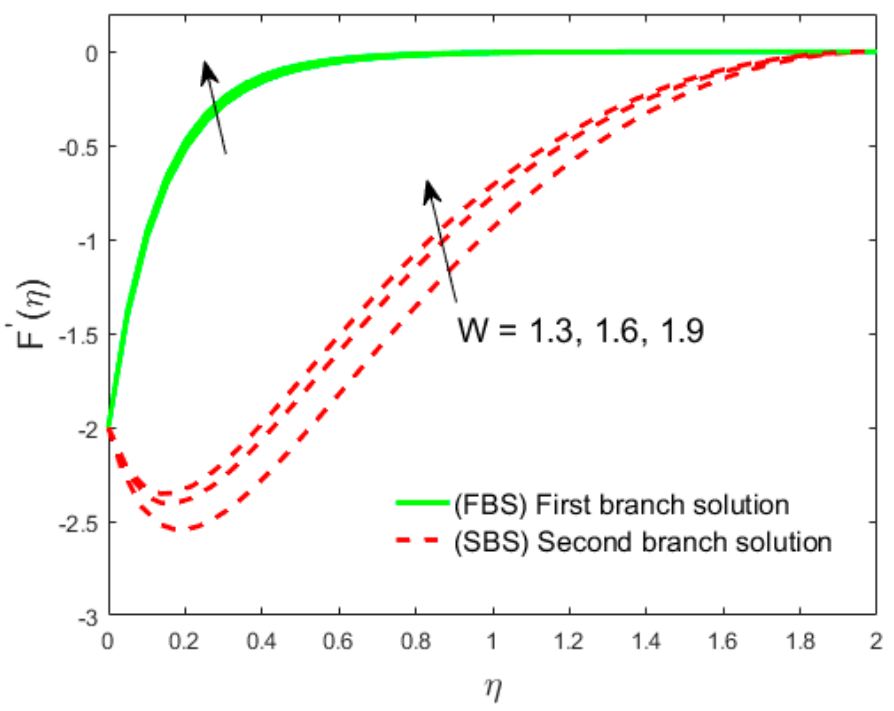

Figure 4. The impact of $W$ on $F^{\prime}(\eta)$.

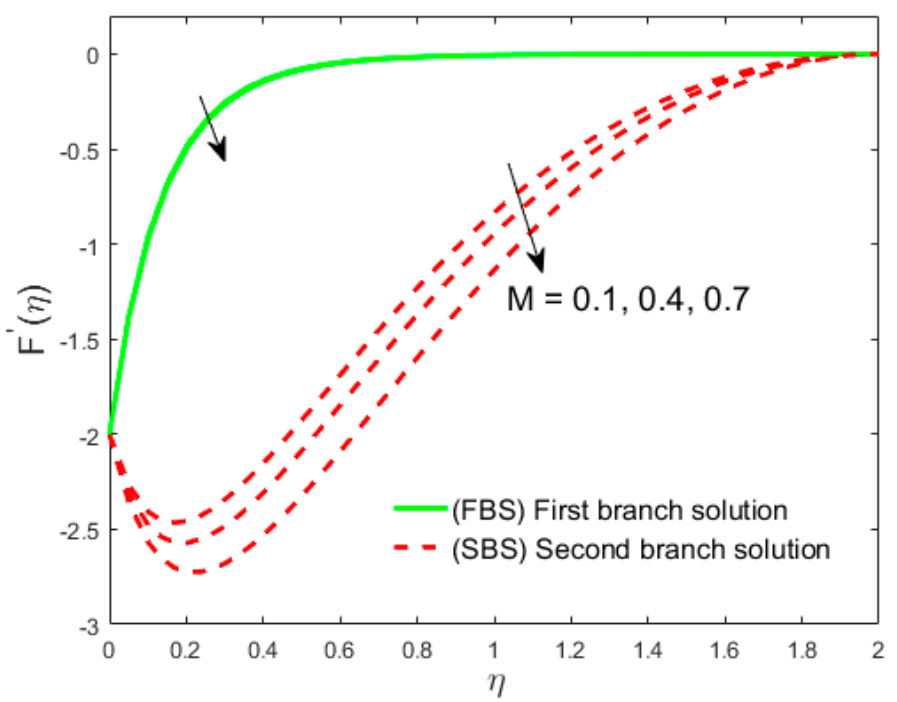

Figure 5. The impact of $M$ on $F^{\prime}(\eta)$. 


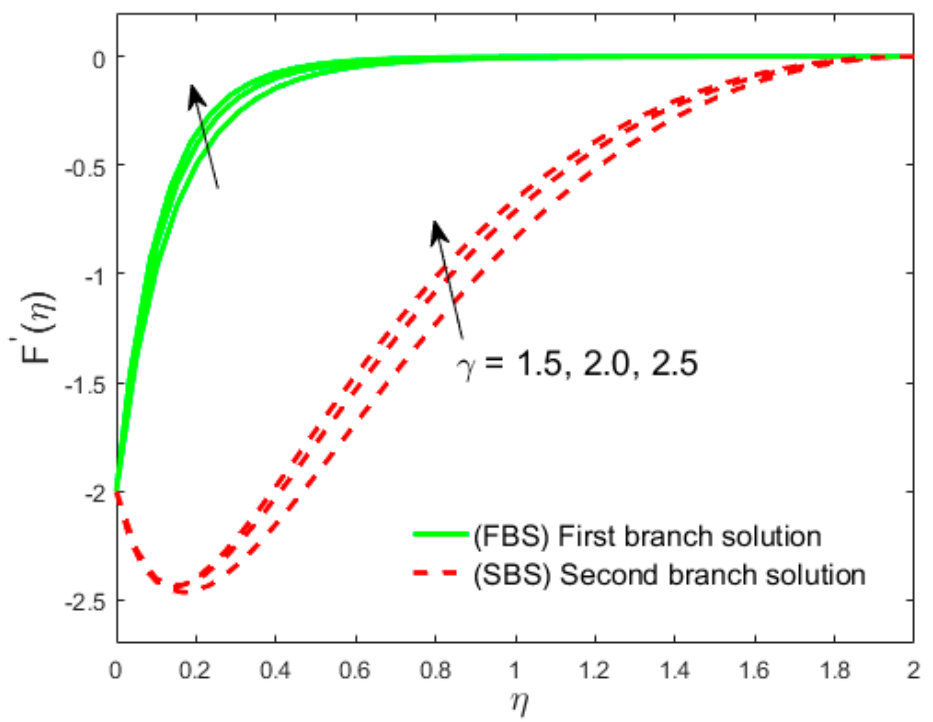

Figure 6. The impact of $\gamma$ on $F^{\prime}(\eta)$.

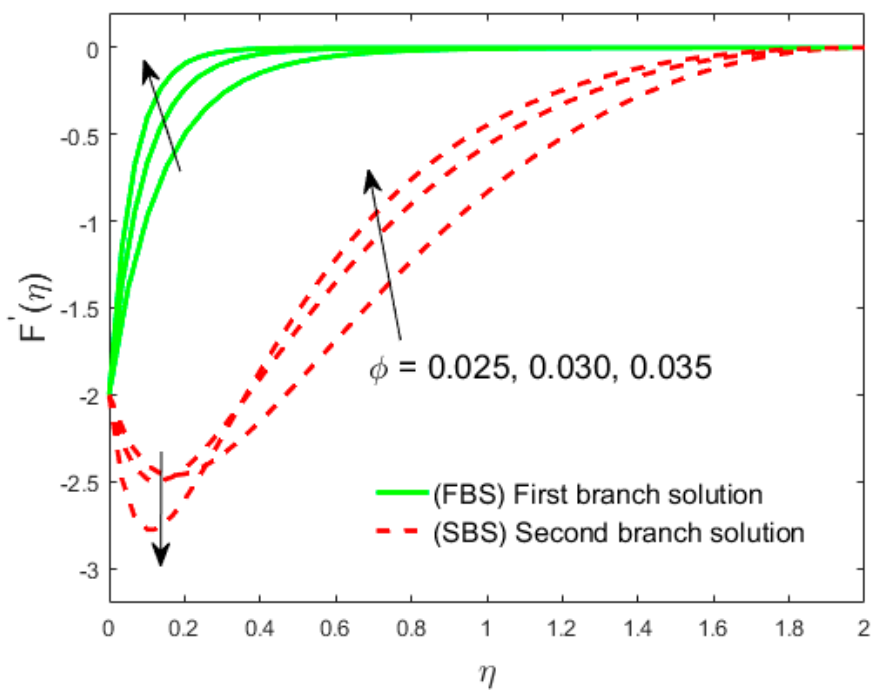

Figure 7. The impact of $\phi$ on $F^{\prime}(\eta)$.

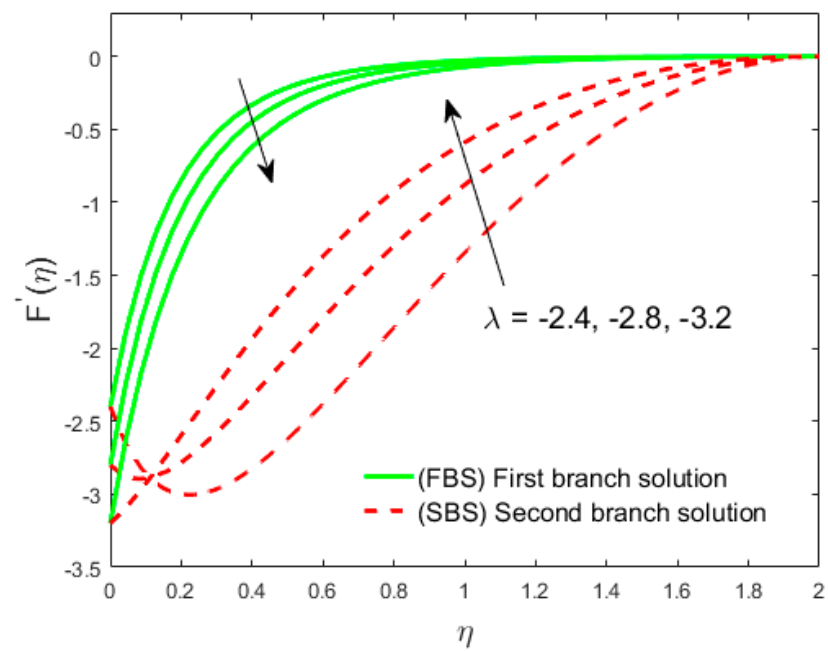

Figure 8. The impact of $\lambda$ on $F^{\prime}(\eta)$. 


\subsection{Temperature Profiles Behavior}

Figures 9-17 explored the behavior of the thermal field due to change in $S_{1}, W, M, \gamma, \phi, R_{d}, \lambda, A_{1}$ and $B_{1}$. Figure 9 is set to scrutinize the impact of suction $S_{1}$ on $\theta(\eta)$. The results explain that the temperature of the liquid and thermal boundary decline due to $S_{1}$ in the UBS and LBS. Physically, the suction generates less resistance in the blood flow, which releases the energy amount due to friction, and consequently, the temperature of blood declines. The stimulus of the curvature parameter $W$ on $\theta(\eta)$ is depicted in Figure 10. It is marked that the distribution of the temperature field is a declining function of $W$ in both solutions. Physically, the decrease in temperature distribution happens due to the translation of a curved surface to a planner surface. The influence of $M$ on $\theta(\eta)$ is illustrated via Figure 11. The temperature depicts a diminishing behavior due to $M$ in the UBS and depicts an uplifting trend in the LBS. Physically, because of the existence of the Lorentz force, less heat energy is generated in the flow, which consequently reduces the blood temperature. Figure 12 explains that due to the Casson parameter, the temperature distribution diminishes in the UBS and LBS. The impact of $\phi$ on $\theta(\eta)$ is shown in Figure 13. It is transparent from this profile that the temperature uplifts due to $\phi$ in both results. The reason behind this is that the gold nanomaterial upsurges the thermal conductivity, which eventually improves the temperature. Figure 14 demonstrates that the temperature is an uplifting function of radiation in the UBS and LBS. The excess of thermal radiation can be explained by the fact that extra heat is captivated through the liquid, and gradually increases the temperature distribution. The influence of the heat phenomenon of source/sink on $\theta(\eta)$ is depicted in Figures 15 and 16. The company of heat source $\left(A_{1}>0, B_{1}>0\right)$ boosts up additional energy within the boundary-layer, which eventually leads to an improvement of the temperature of the liquid (Figure 15), whilst the heat sink $\left(A_{1}<0, B_{1}<0\right)$ sucks up the heat energy from the boundary-layer, which ultimately diminishes the temperature (see Figure 16). Figure 17 portrays the deviation of the shrinking parameter $\lambda$ on the field of temperature distribution $\theta(\eta)$ against the similarity variable $\eta$. The thermal boundary layer and the temperature distribution upsurge in the UBS as well as in the LBS owing to the company of the shrinking parameter.

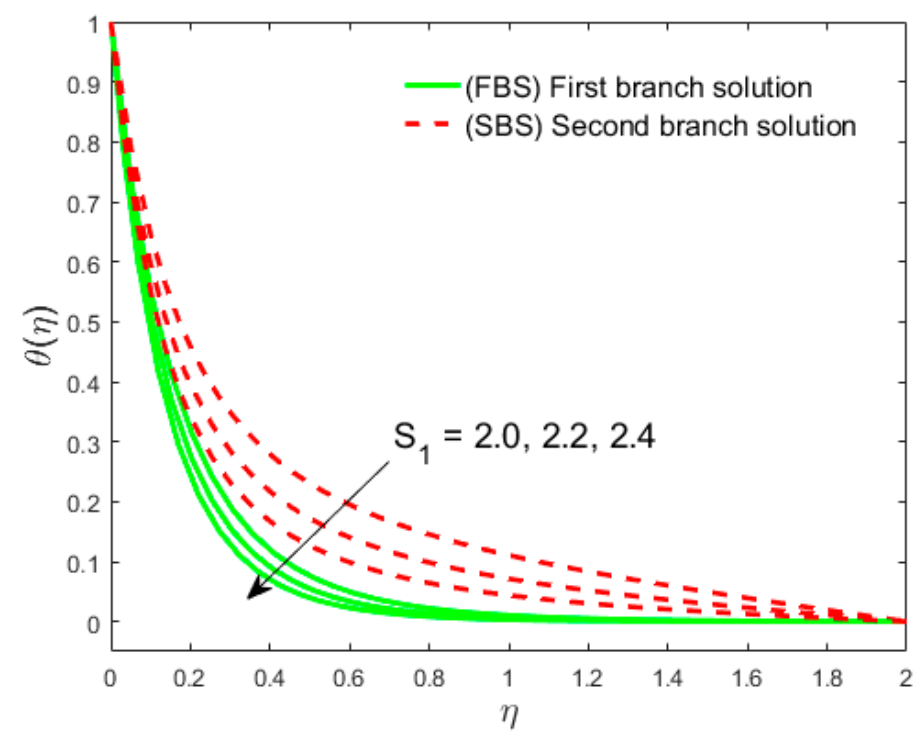

Figure 9. The impact of $S_{1}$ on $\theta(\eta)$. 


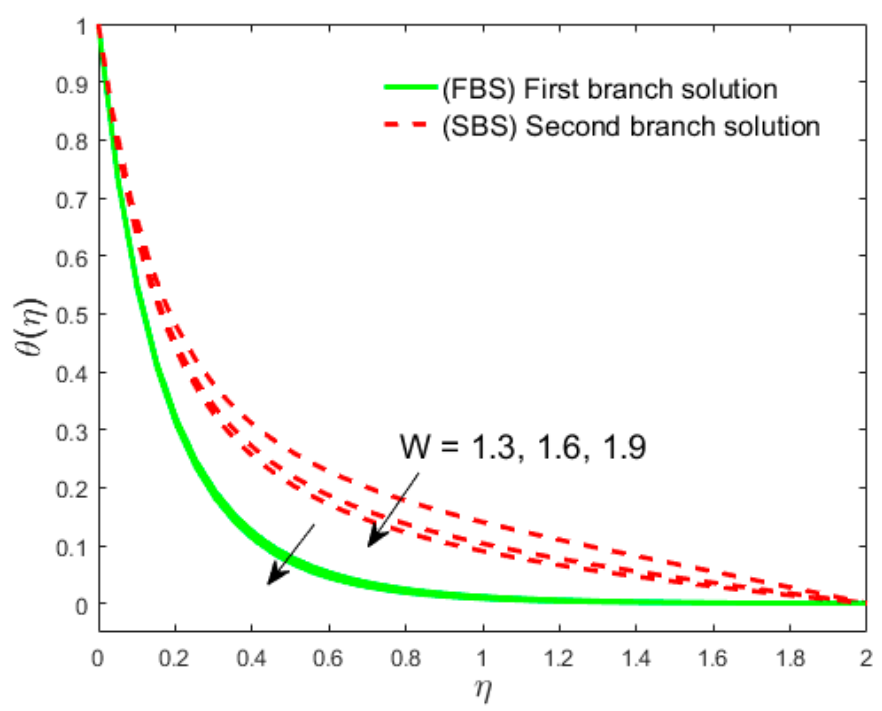

Figure 10. The impact of $W$ on $\theta(\eta)$.

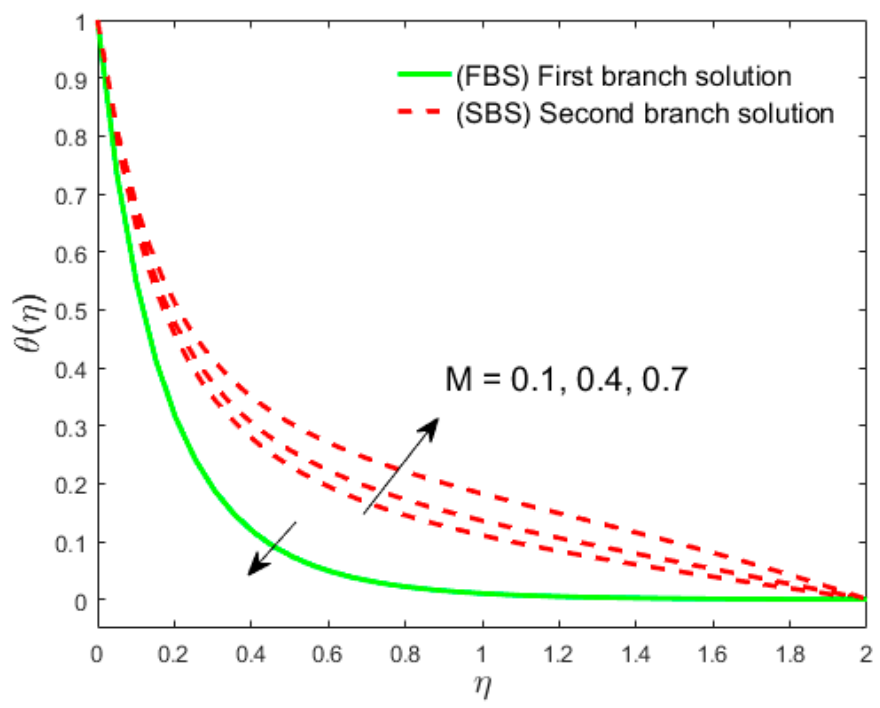

Figure 11. The impact of $M$ on $\theta(\eta)$.

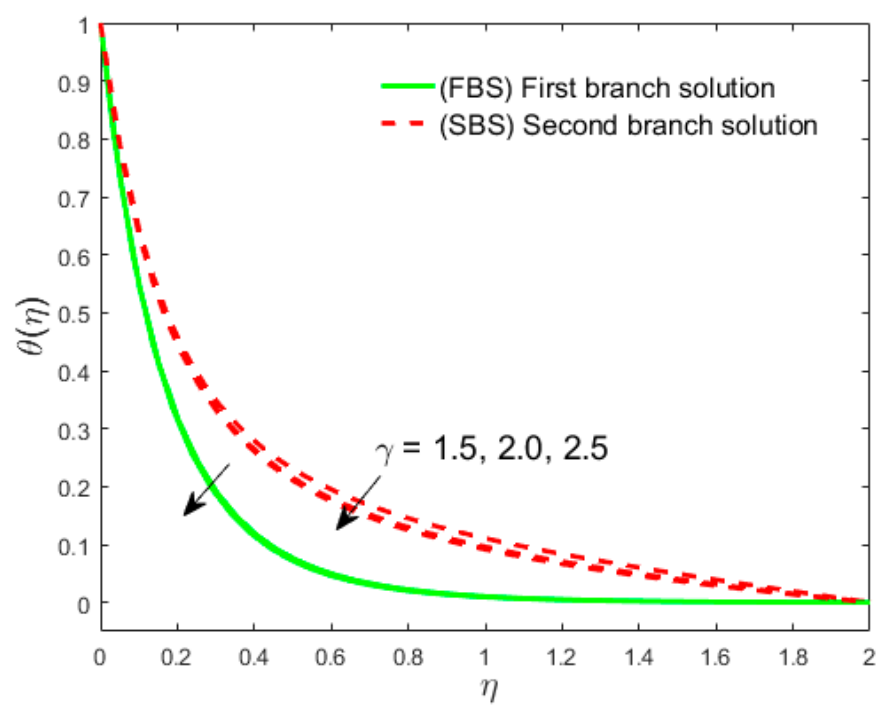

Figure 12. The impact of $\gamma$ on $\theta(\eta)$. 


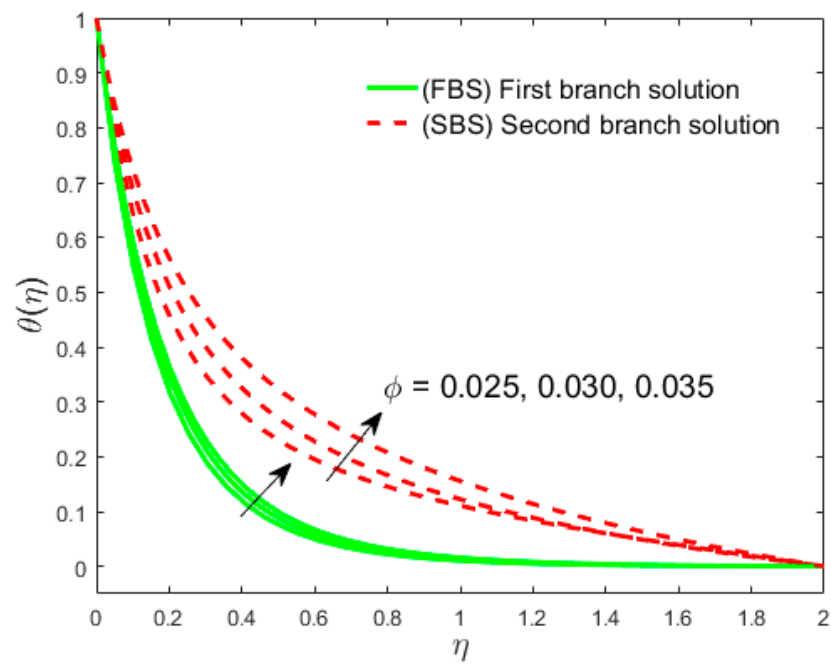

Figure 13. The impact of $\phi$ on $\theta(\eta)$.

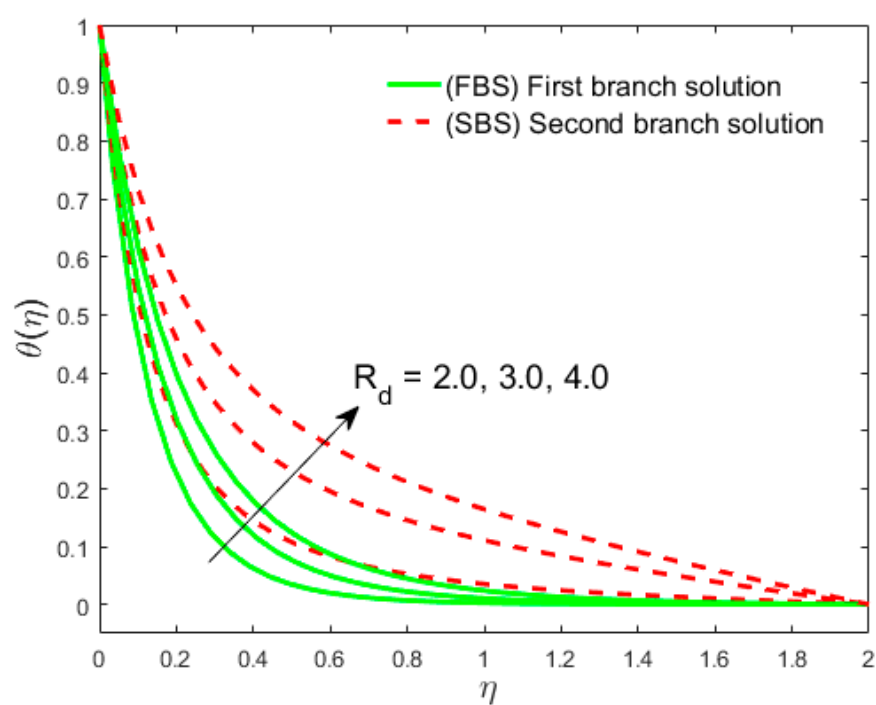

Figure 14. The impact of $R_{d}$ on $\theta(\eta)$.

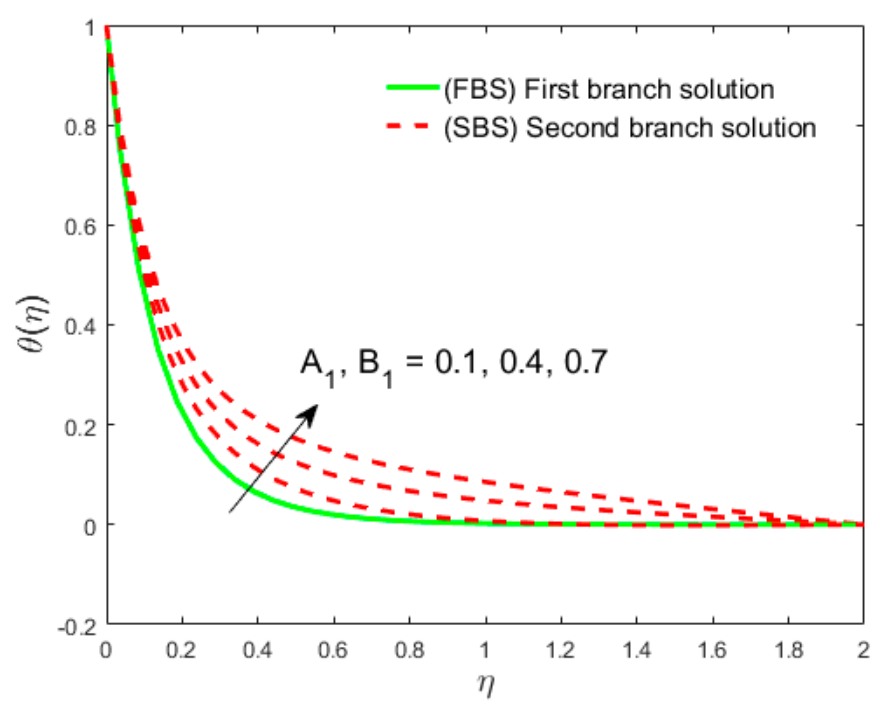

Figure 15. The impact of $A_{1}=B_{1}>0$ on $\theta(\eta)$. 


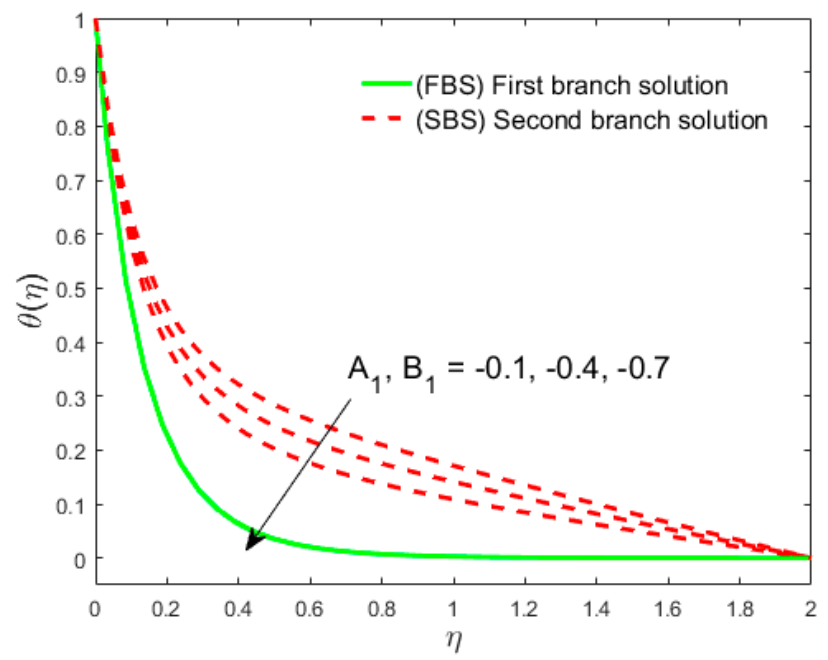

Figure 16. Influence of $A_{1}=B_{1}<0$ on $\theta(\eta)$.

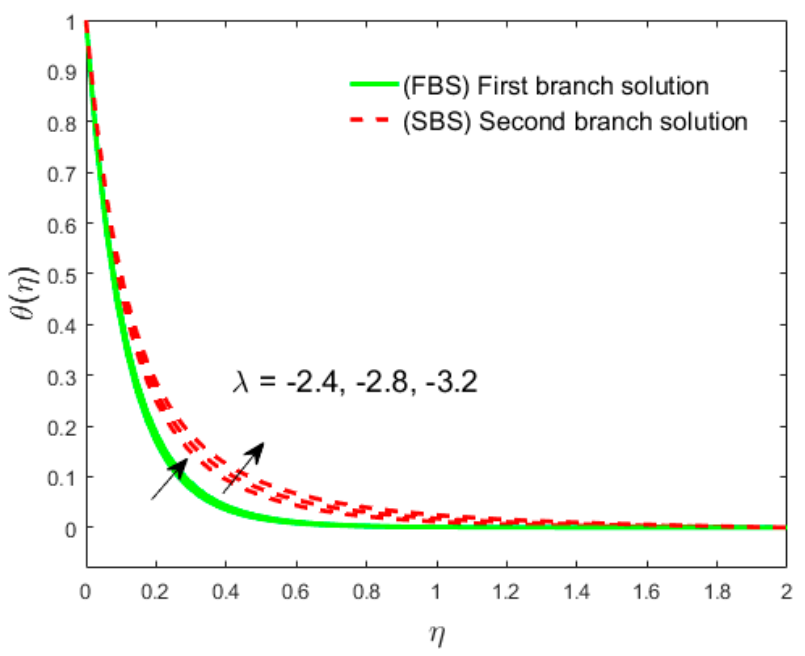

Figure 17. Influence of $\lambda$ on $\theta(\eta)$.

\subsection{The Behavior of the Skin Factor and Local Heat Transfer Rate}

The graphical solutions for the skin friction and rate of heat transfer under the deviation of distinct constraints $W, \gamma, \phi$ against $\lambda$ (stretched/shrinking parameter) are illustrated in Figures 18-23. Figure 18 explains that the solution of the upper branch classifies an improvement in the friction factor due to $W$; however, the solution of the lower branch sights the decrement in friction factor. This behavior can suggest the flow via severance where the pattern of small wake could diminish the surface shear-stress with the curvature parameter. Hence, the amount of friction factor declines. Figure 19 displays that the heat transfer rate upsurges with $W$ in the dashed and solid solutions. Moreover, the results of the local heat transfer rate are optimistic for the heat conductivity to be transferred from a hot surface to a cold solution. Multiple results exist in the region of $\lambda_{c}<\lambda \leq-3.88009$, a single result for $\lambda=\lambda_{c}$ and no results for $\lambda<\lambda_{c}$. It is transparent from these portraits that the critical values $\left|\lambda_{c}\right|$ augment identifying that the curvature parameter impediments the separation. Figure 20 depicts that, owing to the Casson parameter $\gamma$, the friction factor initially decreases and then climbs in the UBS after a certain value of $\lambda$ and declines in the dashed LBS. However, the heat transfer rate (Figure 21) upsurges owing to $\gamma$ in the stable outcomes and shrinks in the unstable outcomes. These sketches also suggest that the company of the Casson parameter diminished the BLF separation. Figures 22 and 23 illustrate the deviation of $\phi$ against $\lambda$ on the skin friction and the rate of heat transfer. The behavior of the skin factor (Figure 21) initially decreases and then uplifts 
an improve from 0.02 onward during the upper solution, and decays throughout the lower solution. The explanation is that the thermal conductivity of the gold nanoparticle gets higher, and there is a suspension of additional particles in the blood flow. A similar trend is scrutinized for the heat transfer rate, as depicted in Figure 23.

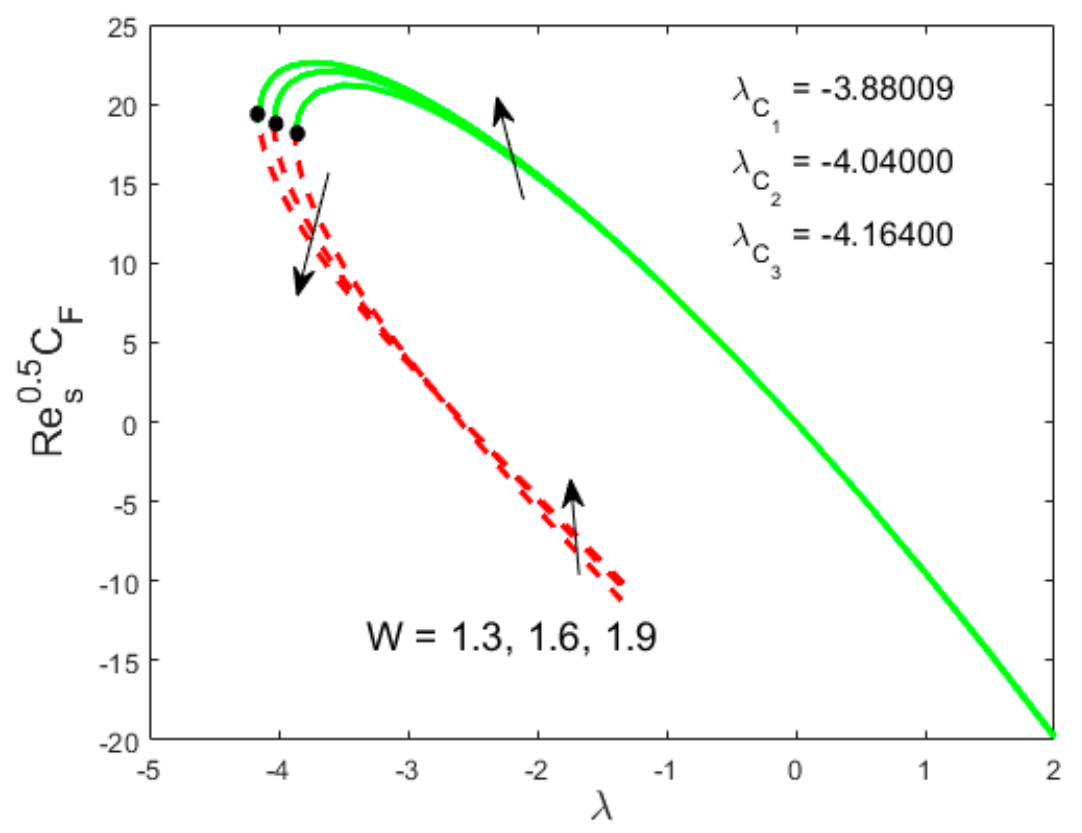

Figure 18. Influence of $W$ on $\operatorname{Re}_{\mathrm{s}}^{0.5} C_{F}$ versus $\lambda$.

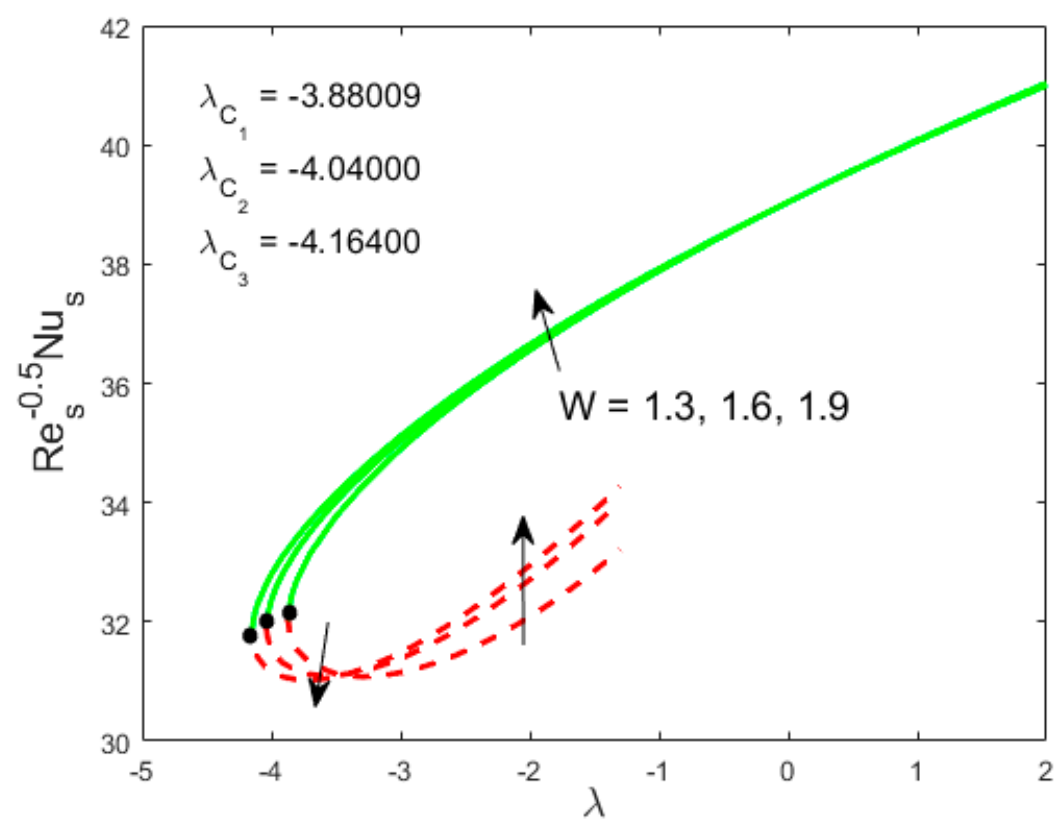

Figure 19. Influence of $W$ on $\operatorname{Re}_{\mathrm{s}}^{-0.5} N u_{\mathrm{s}}$ versus $\lambda$. 


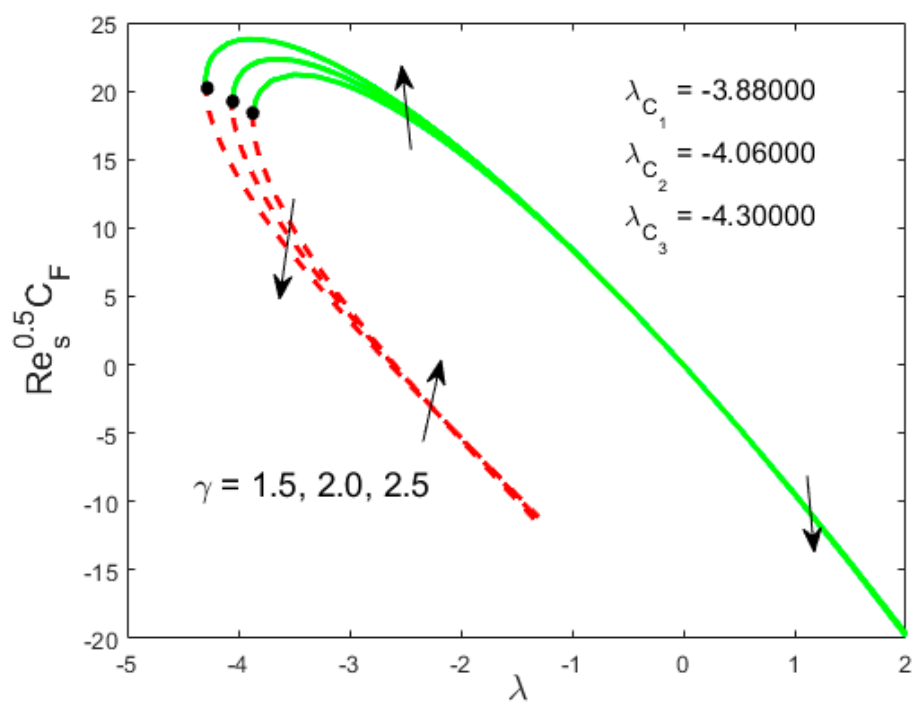

Figure 20. The impact of $\gamma$ on $\operatorname{Re}_{\mathrm{s}}^{0.5} C_{F}$ versus $\lambda$.

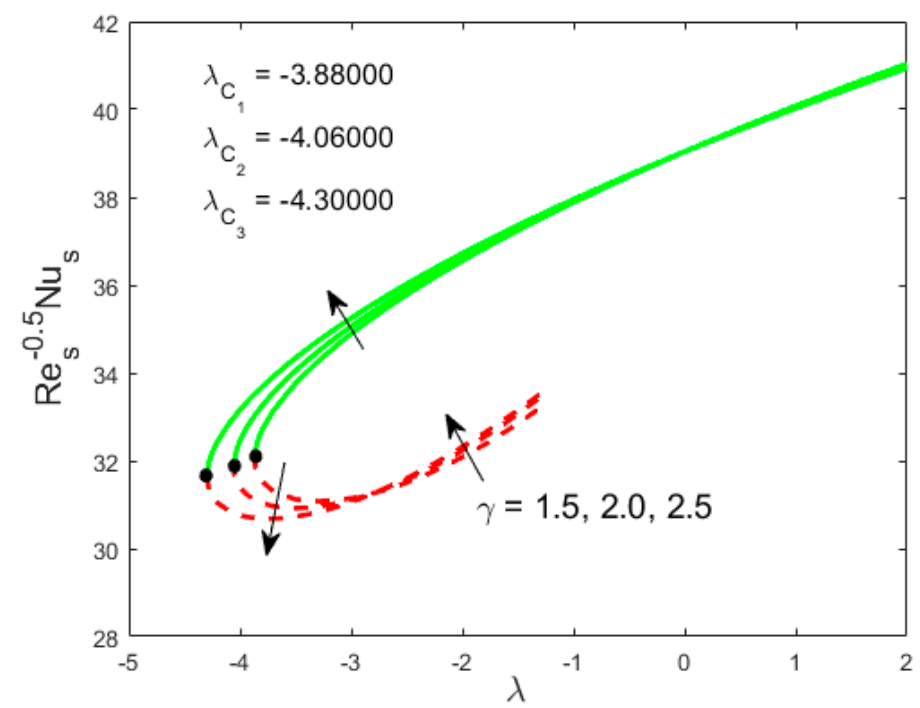

Figure 21. The impact of $\gamma$ on $\operatorname{Re}_{\mathrm{s}}^{-0.5} N u_{\mathrm{s}}$ versus $\lambda$.

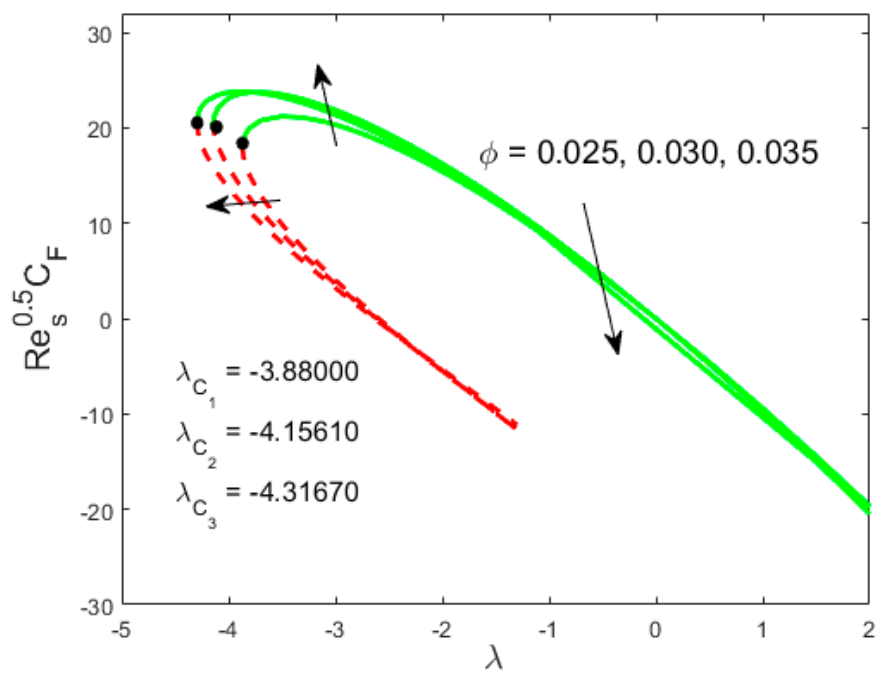

Figure 22. Influence of $\phi$ on $\operatorname{Re}_{\mathrm{s}}^{0.5} C_{F}$ versus $\lambda$. 


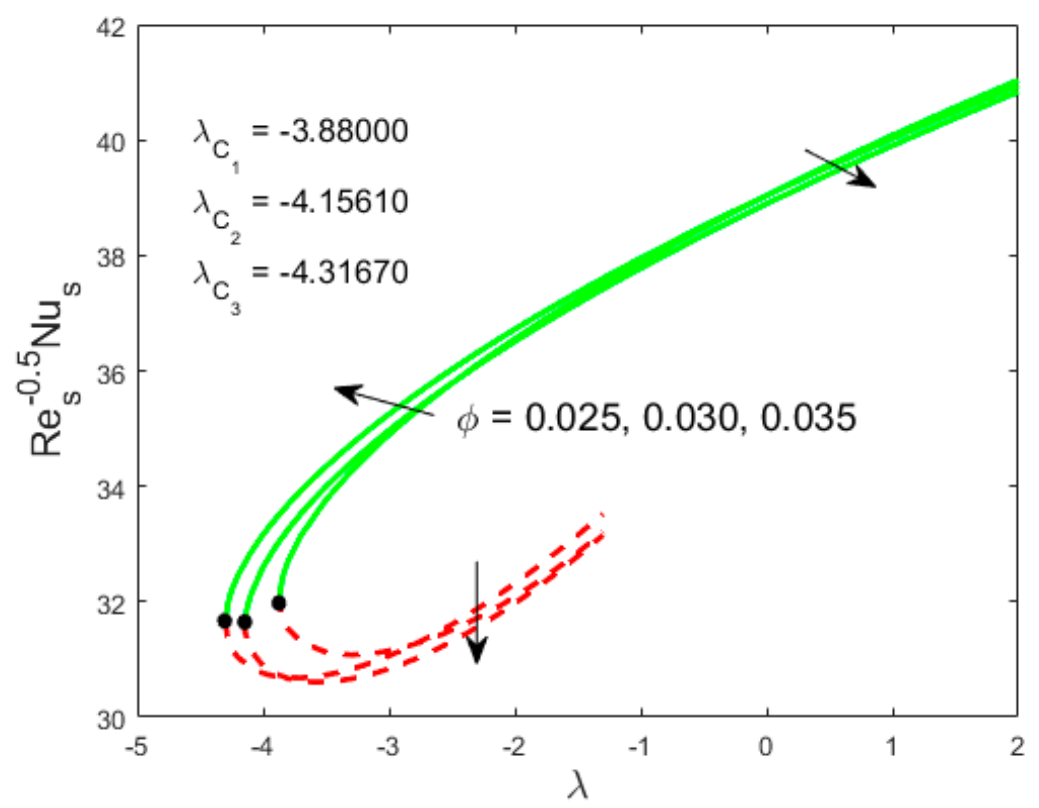

Figure 23. Influence of $\phi$ on $\operatorname{Re}_{\mathrm{s}}^{-0.5} N u_{\mathrm{s}}$ versus $\lambda$.

\section{Conclusions}

In the current exploration, the problem of magneto radiative blood flow comprising gold particles via a moving curved/flat sheet with a non-uniform heat sink/source has been examined. The leading PDEs are renovated into nonlinear ODEs utilizing appropriate variables and then worked out through the bvp4c solver. The finishing remarks with regard to the current research are listed as follows:

- The velocity gradient uplifts owing to $S_{1}$ in the UBS and diminishes in the LBS; however, the temperature field declines in both branches of outcomes.

- $\theta(\eta)$ reduces due to $W$ in both the lower and upper solutions, while $F^{\prime}(\eta)$ upsurges.

- The magnetic number can be exercised to control the blood flow as well as the temperature by regulating the magnetic intensity.

- Uplifting curvature leads to an enhancement in the heat transfer in both solutions and leads to an increase in the skin factor behavior in the UBS.

- Due to $\phi$ and $\gamma$, the skin factor behavior of the flow of fluid in the beginning decelerates and then uplifts after a certain value of $\lambda$.

- The nanoparticle volume fraction accelerates the viscosity of blood as well as the thermal conductivity.

- The Casson gold blood parameter shrinks the thickness of the velocity boundary-layer owing to rapid enhancement in the plastic dynamics' viscosity.

- The radiation parameter augments the temperature distribution in both solutions.

- The heat source upsurges the temperature of the fluid, while the heat sink decelerates the temperature in both solutions.

- The Casson gold blood parameter in the UBS accelerates the heat transfer and, on the other hand, the LBS is decline.

- The heat transfer rate initially upsurges and then declines due to $\phi$ in the case of the UBS, whilst the reverse behavior is noted in the LBS.

Author Contributions: Conceptualization, A.Z.; data curation, U.K.; formal analysis, A.S. and U.K.; funding acquisition, D.B.; methodology, U.K.; resources, A.S.; supervision, A.Z.; writing-review and editing, E.-S.M.S. and D.B. All authors have read and agreed to the published version of the manuscript.

Funding: The authors are grateful to King Saud University for funding this work through Researchers supporting Project number (RSP-2020/33). 
Acknowledgments: Researchers supporting Project number (RSP-2020/33), King Saud University, Riyadh, Saudi Arabia.

Conflicts of Interest: The authors declare no conflict of interest.

\section{Nomenclature}

\begin{tabular}{|c|c|}
\hline$a_{1}$ & positive constant \\
\hline$A_{1}, B_{1}$ & space and temperature-dependent heat source/sink \\
\hline$B_{0}$ & the intensity of the magnetic field \\
\hline$C_{F}$ & skin friction coefficients \\
\hline$c_{p}$ & specific heat \\
\hline$F$ & dimensionless velocities \\
\hline$k^{*}$ & mean proportion constant \\
\hline$k_{n f}$ & nanofluid thermal conductivity \\
\hline$M$ & magnetic parameter \\
\hline$N u_{s}$ & Nusselt number \\
\hline$p$ & pressure \\
\hline $\operatorname{Pr}$ & Prandtl number \\
\hline$q^{\prime \prime \prime}$ & erratic heat sink/source \\
\hline$q_{r}$ & radiative heat flux \\
\hline$q_{w}$ & wall heat flux \\
\hline$R$ & radius \\
\hline$R_{d}$ & radiation parameter \\
\hline$r^{a}$ & modified space variable \\
\hline $\operatorname{Re}_{s}$ & local Reynolds number \\
\hline$S_{1}$ & suction \\
\hline$T_{1}$ & temperature \\
\hline$T_{\infty}$ & free-stream temperature \\
\hline$T_{w}$ & wall temperature \\
\hline$v_{0}$ & mass-flux velocity \\
\hline$\left(u_{1}, v_{1}\right)$ & velocity components \\
\hline$(r, s)$ & curvilinear coordinates \\
\hline W & curvature parameter \\
\hline \multicolumn{2}{|c|}{ Greek symbols } \\
\hline$\gamma$ & Casson parameter \\
\hline$\lambda$ & stretching/shrinking parameter \\
\hline$\mu_{n f}$ & nanofluid dynamic viscosity \\
\hline$\phi$ & volume fractions of nanoparticle \\
\hline$\theta$ & dimensionless temperature \\
\hline$\Gamma$ & time constant \\
\hline$v_{f}$ & kinematic viscosity of a base fluid \\
\hline$\rho_{n f}$ & nanofluid density \\
\hline$\left(\rho c_{p}\right)_{n f}$ & heat capacitance of nanofluid \\
\hline$\sigma^{*}$ & Stefan-Boltzmann constant \\
\hline$\sigma_{n f}$ & the electrical conductivity of nanofluid \\
\hline$\tau_{s}$ & shear stress \\
\hline$\psi$ & stream function \\
\hline$\eta$ & similarity variable \\
\hline \multicolumn{2}{|c|}{ Subscripts } \\
\hline$s_{1}$ & solid nanoparticle \\
\hline$N f$ & nanofluid \\
\hline$F$ & base fluid \\
\hline Supersc & \\
\hline
\end{tabular}




\section{References}

1. Huang, X.; El-Sayed, M.A. Gold nanoparticles: Optical properties and implementations in cancer diagnosis and photothermal therapy. J. Adv. Res. 2010, 1, 13-28. [CrossRef]

2. Kumar, P.K.; Paul, W.; Sharma, C.P. Green synthesis of gold nanoparticles with Zingiberofficinale extract: Characterization and blood compatibility. Process Biochem. 2011, 46, 2007-2013.

3. Hatami, M.; Hatami, J.; Ganji, D. Computer simulation of MHD blood conveying gold nanoparticles as a third grade non-Newtonian nanofluid in a hollow porous vessel. Comput. Methods Programs Biomed. 2014, 113, 632-641. [CrossRef] [PubMed]

4. Hamzehnezhad, A.; Fakour, M.; Ganji, D.D.; Rahbari, A. Heat transfer and fluid flow of blood flow containing nanoparticles through porous blood vessels with magnetic field. Math. Biosci. 2017, 283, 38-47.

5. Elnaqeeb, T.; Mekheimer, K.S.; Alghamdi, F. Cu-blood flow model through a catheterized mild stenotic artery with a thrombosis. Math. Biosci. 2016, 282, 135-146. [CrossRef]

6. Mekheimer, K.S.; Elnaqeeb, T.; El Kot, M.A.; Alghamdi, F. Simultaneous effect of magnetic field and metallic nanoparticles on a micropolar fluid through an overlapping stenotic artery: Blood flow model. Phys. Essays 2016, 29, 272-283. [CrossRef]

7. Sheikholeslami, M.; Shamlooei, M. Magnetic source influence on nanofluid flow in porous medium considering shape factor effect. Phys. Lett. A 2017, 381, 3071-3078. [CrossRef]

8. Maleki, H.; Alsarraf, J.; Moghanizadeh, A.; Hajabdollahi, H.; Safaei, M.R. Heat transfer and nanofluid flow over a porous plate with radiation and slip boundary conditions. J. Cent. South. Univ. 2019, 26, 1099-1115. [CrossRef]

9. Maleki, H.; Safaei, M.R.; Togun, H.; Dahari, M. Heat transfer and fluid flow of pseudo-plastic nanofluid over a moving permeable plate with viscous dissipation and heat absorption/generation. J. Therm. Anal. Calorim. 2018, 135, 1643-1654. [CrossRef]

10. Maleki, H.; Safaei, M.R.; Alrashed, A.A.A.A.; Kasaeian, A. Flow and heat transfer in non-Newtonian nanofluids over porous surfaces. J. Therm. Anal. Calorim. 2018, 135, 1655-1666. [CrossRef]

11. Harris, J. Rheology and Non-Newtonian Flow; Longman Publishing Group: Harlow, UK, 1977.

12. Bird, R.B.; Curtis, C.F.; Armstrong, R.C.; Hassager, O. Dynamics of Polyometric Liquids; Wiley: Hoboken, NJ, USA, 1987.

13. Mukhopadhyay, S.; Mondal, I.C.; Chamkha, A.J. Casson fluid flow and heat transfer past a symmetric wedge. Heat Transf. Asian Res. 2013, 42, 665-675. [CrossRef]

14. Rashidi, M.; Rastegari, M.T.; Asadi, M.; Bég, O.A. A Study of Non-Newtonian Flow and Heat Transfer Over a Non-Isothermal Wedge Using the Homotopy Analysis Method. Chem. Eng. Commun. 2012, 199, 231-256. [CrossRef]

15. Jamalabadi, M.Y.A.; DaqiqShirazi, M.; Nasiri, H.; Safaei, M.R.; Nguyen, T.K. Modeling and analysis of biomagnetic blood Carreau fluid flow through a stenosis artery with magnetic heat transfer: A transient study. PLoS ONE 2018, 13, e0192138. [CrossRef]

16. Amiri, M.H.; Keshavarzi, A.; Karimipour, A.; Bahiraei, M.; Goodarzi, M.; Esfahani, J.A. A 3-D numerical simulation of non-Newtonian blood flow through femoral artery bifurcation with a moderate arteriosclerosis: Investigating Newtonian/non-Newtonian flow and its effects on elastic vessel walls. Heat Mass Transf. 2019, 55, 2037-2047. [CrossRef]

17. Shafiq, A.; Sindhu, T. Statistical study of hydromagnetic boundary layer flow of Williamson fluid regarding a radiative surface. Results Phys. 2017, 7, 3059-3067. [CrossRef]

18. Mabood, F.; Shafiq, A.; Hayat, T.; Abelman, S. Radiation effects on stagnation point flow with melting heat transfer and second order slip. Results Phys. 2017, 7, 31-42. [CrossRef]

19. Abbas, Z.; Naveed, M.; Sajid, M. Hydromagnetic slip flow of nanofluid over a curved stretching surface with heat generation and thermal radiation. J. Mol. Liq. 2016, 215, 756-762. [CrossRef]

20. Acharya, N.; Mabood, F. On the hydrothermal features of radiative $\mathrm{Fe}_{3} \mathrm{O}_{4}$-graphene hybrid nanofluid flow over a slippery bended surface with heat source/sink. J. Therm. Anal. Calorim. 2020, 1-17. [CrossRef]

21. Mintsa, H.A.; Nguyen, C.T.; Roy, G. New temperature dependent thermal conductivity data of water based nanofluids. In Proceedings of the 5th IASME/WSEAS international Conference on Heat Transfer, Thermal Engineering and Environment, Athens, Greece, 25-27 August 2007; Volume 290, pp. 25-27. 
22. Afshari, A.; Akbari, M.; Toghraie, D.; Yazdi, M.E. Experimental investigation of rheological behavior of the hybrid nanofluid of MWCNT-alumina/water (80\%)-ethylene-glycol (20\%). J. Therm. Anal. Calorim. 2018, 132, 1001-1015. [CrossRef]

23. Makinde, O.D.; Animasaun, I.; Animasaun, I.L. Thermophoresis and Brownian motion effects on MHD bioconvection of nanofluid with nonlinear thermal radiation and quartic chemical reaction past an upper horizontal surface of a paraboloid of revolution. J. Mol. Liq. 2016, 221, 733-743. [CrossRef]

24. Weidman, P.; Kubitschek, D.; Davis, A. The effect of transpiration on self-similar boundary layer flow over moving surfaces. Int. J. Eng. Sci. 2006, 44, 730-737. [CrossRef]

25. Zaib, A.; Khan, U.; Wakif, A.; Zaydan, M. Numerical Entropic Analysis of Mixed MHD Convective Flows from a Non-Isothermal Vertical Flat Plate for Radiative Tangent Hyperbolic Blood Biofluids Conveying Magnetite Ferroparticles: Dual Similarity Solutions. Arab. J. Sci. Eng. 2020, 45, 1-20. [CrossRef]

26. Sharma, R.; Ishak, A.; Pop, I. Stability analysis of magnetohydrodynamic stagnation-point flow toward a stretching/shrinking sheet. Comput. Fluids 2014, 102, 94-98. [CrossRef]

27. Roşca, A.V.; Pop, I. Flow and heat transfer over a vertical permeable stretching/shrinking sheet with a second order slip. Int. J. Heat Mass Transf. 2013, 60, 355-364. [CrossRef]

28. Chato, J.C. Heat Transfer to Blood Vessels. J. Biomech. Eng. 1980, 102, 110-118. [CrossRef] [PubMed]

29. Kafoussias, N.E.; Tzirtzilakis, E. Biomagnetic fluid flow over a stretching sheet with non linear temperature dependent magnetization. Z. Angew. Math. Phys. 2003, 54, 551-565. [CrossRef]

30. Sanni, K.; Asghar, S.; Jalil, M.; Okechi, N. Flow of viscous fluid along a nonlinearly stretching curved surface. Results Phys. 2017, 7, 1-4. [CrossRef]

31. Abbas, Z.; Naveed, M.; Sajid, M. Heat transfer analysis for stretching flow over a curved surface with magnetic field. J. Eng. Thermophys. 2013, 22, 337-345. [CrossRef]

32. Koriko, O.K.; Animasaun, I.; Mahanthesh, B.; Saleem, S.; Sarojamma, G.; Sivaraj, R. Heat transfer in the flow of blood-gold Carreau nanofluid induced by partial slip and buoyancy. Heat Transf. Asian Res. 2018, 47, 806-823. [CrossRef]

(C) 2020 by the authors. Licensee MDPI, Basel, Switzerland. This article is an open access article distributed under the terms and conditions of the Creative Commons Attribution (CC BY) license (http://creativecommons.org/licenses/by/4.0/). 